\title{
EKMAN BOUNDARY LAYERS IN ROTATING FLUIDS
}

\author{
Jean-Yves Chemin $^{1}$, Benoît Desjardins ${ }^{2}$, Isabelle Gallagher ${ }^{1}$ and \\ EMMANUEL GRENIER ${ }^{3}$
}

\begin{abstract}
In this paper, we investigate the problem of fast rotating fluids between two infinite plates with Dirichlet boundary conditions and "turbulent viscosity" for general $L^{2}$ initial data. We use dispersive effect to prove strong convergence to the solution of the bimensionnal Navier-Stokes equations modified by the Ekman pumping term.
\end{abstract}

Mathematics Subject Classification. 35Q30, 35Q35, 76U05.

Received January 7, 2002.

\section{INTRODUCTION}

In this paper, we investigate the problem of fast rotating viscous fluids between two plates with Dirichlet boundary conditions. We present the model with so called "turbulent" viscosity. More precisely, we shall study the limit when $\varepsilon$ tends to 0 of the system involving the velocity field $u^{\varepsilon}$ and the pressure $p^{\varepsilon}$

$$
\left(N S C_{\varepsilon}\right)\left\{\begin{aligned}
\Delta_{t} u^{\varepsilon}+\operatorname{div}\left(u^{\varepsilon} \otimes u^{\varepsilon}\right)-\nu \Delta_{h} u^{\varepsilon}-\beta \varepsilon \Delta_{3}^{2} u^{\varepsilon}+\frac{e_{3} \times u^{\varepsilon}}{\varepsilon} & =-\nabla p^{\varepsilon} \\
\operatorname{div} u^{\varepsilon} & =0 \\
u_{\mid \Delta \Omega}^{\varepsilon} & =0 \\
u_{\mid t=0}^{\varepsilon} & =u_{0}^{\varepsilon} \in L^{2}(\Omega)
\end{aligned}\right.
$$

where $\left.\Omega=\mathbf{R}^{2} \times\right] 0,1\left[\right.$. We shall use the following notation: if $u$ is a vector on $\mathbf{R}^{3}$ we state $u=\left(u^{1}, u^{2}, u^{3}\right)$ $=\left(u^{h}, u^{3}\right)$, and we will note $\Delta_{h}=\partial_{1}^{2}+\partial_{2}^{2}$. Moreover, if $f$ is a function on $\Omega, \mathcal{F} f$ (and also $\widehat{f}$ ) will denote the Fourier transform with respect to the horizontal variable $x_{h}$. Finally in all that follows, the space $L^{2}$ (with no argument) will denote the space $L^{2}(\Omega)$.

These equations arise in physical contexts when one studies oceanic or atmospheric motions. In general geophysical flows can be considered as incompressible, and with the length and time scales considered, the rotation of the Earth can not be neglected and on the contrary plays a major role. Basically under high rotation, a three dimensional fluid tends to behave like a two dimensional one, and to become invariant in the direction of the rotation. This well known phenomena, called Taylor Proudman theorem is detailed in the monographs [7] and [11]. The part of the initial data which depends on the vertical component creates waves,

Keywords and phrases: Navier-Stokes equations, rotating fluids, Strichartz estimates.

1 Centre de Mathématiques de l'École Polytechnique, UMR 7640 du CNRS, 91128 Palaiseau Cedex, France;

e-mail: Isabelle.Gallagher@math.polytechnique.fr

2 CEA, BP. 12, 91680 Bruyères-le-Châtel, France.

3 UMPA, UMR 5669 du CNRS, ENS Lyon, 46 allée d'Italie, 69364 Lyon Cedex 07, France. 
called inertial waves, which propagate with a high speed. Moreover, keeping in mind this anisotropy, it is usual to consider an anisotropic viscosity like in $\left(N S C_{\varepsilon}\right)$, the horizontal "turbulent" diffusion being larger than the vertical one. In the periodic case we refer to $[1,6,8]$ for mathematical studies. In the presence of horizontal boundaries, boundary layers called Ekman layers appear to match the Dirichlet boundary condition with the tendency of the fluid to become independent on the third variable $x_{3}$. It turns out that these layers are always stable in the case of anisotropic viscosity. For previous studies we refer to [5] and [9]. The aim of this paper is to combine the study of these layers with the study of the dispersion of the inertial waves (we consider an unbounded domain).

In the sequel, we shall assume that $u_{0}^{\varepsilon} \cdot n=u_{0}^{\varepsilon, 3}=0$ on $\Delta \Omega$, and $\operatorname{div} u_{0}^{\varepsilon}=0$. This condition $u_{0}^{\varepsilon, 3}=0$ implies the following fact: for any $L^{2}$ divergence free vector field $u$, the function $\partial_{3} u^{3}$ is $L^{2}(] 0,1[)$ with respect to the variable $x_{3}$ with value in $H^{-1}\left(\mathbf{R}^{2}\right)$. Hence by integration, we get

$$
u^{3}\left(x_{h}, 1\right)-u^{3}\left(x_{h}, 0\right)=-\int_{0}^{1} \operatorname{div}_{h} u^{h}\left(x_{h}, x_{3}\right) \mathrm{d} x_{3}=0 .
$$

So the vertical mean value of the horizontal part on the vector field is divergence free as a vector field on $\mathbf{R}^{2}$. Of course, a quantity will play a key role: this is (twice) the energy defined by

$$
\mathcal{E}_{\varepsilon}(v)(t) \stackrel{\text { def }}{=}\|v(t)\|_{L^{2}}^{2}+2 \nu_{h} \int_{0}^{t}\left\|\nabla^{h} v\left(t^{\prime}\right)\right\|_{L^{2}}^{2} \mathrm{~d} t^{\prime}+2 \beta \varepsilon \int_{0}^{t}\left\|\partial_{3} v\left(t^{\prime}\right)\right\|_{L^{2}}^{2} \mathrm{~d} t^{\prime} .
$$

The reason why we choose the vertical viscosity of the form $\beta \varepsilon$ will be explained in Section 1 where we shall recall the properties of the "well prepared" case. The relation between this choice and the Ekman pumping will be studied in detail in that next section. First of all we shall study the linear problem in the well prepared case, which means that the function $u_{0}$ does not depend on the vertical variable $x_{3}$. Then we shall investigate the more delicate case when the initial data $u_{0}$ does actually depend on the vertical variable $x_{3}$. We shall compute explicitly approximate solutions for the linear problem.

To study the real problem we have to deal with non linear terms. In the well prepared case, we have strong convergence in $L^{2}$, as well as in the case when the domain is $\left.\mathbf{T}^{2} \times\right] 0,1[$ (see [9]). The precise theorem proved in $[9]$ is the following:

Theorem 1. Let $u^{\varepsilon}$ be a family of weak solutions of $\left(N S C_{\varepsilon}\right)$ associated with a family of initial data $u_{0}^{\varepsilon}$ of divergence free vector fields such that

$$
\lim _{\varepsilon \rightarrow 0} u_{0}^{\varepsilon}=\left(\bar{u}_{0}, 0\right) \quad \text { in } \quad L^{2}
$$

where $\bar{u}_{0}$ is a divergence free vector field in $L^{2}\left(\mathbf{R}^{2}\right)$. Denoting by $\bar{u}$ the global solution of the two dimensional Navier-Stokes type equations

$$
\left(N S E_{\nu, \beta}\right)\left\{\begin{aligned}
\Delta_{t} \bar{u}+\operatorname{div}_{h}(\bar{u} \otimes \bar{u})-\nu \Delta_{h} \bar{u}+\sqrt{2 \beta} \bar{u} & =-\nabla^{h} \bar{p} \\
\operatorname{div}_{h} \bar{u} & =0 \\
\bar{u}_{\mid t=0} & =\bar{u}_{0},
\end{aligned}\right.
$$

we have

$$
\left\|u^{\varepsilon}-(\bar{u}, 0)\right\|_{L^{\infty}\left(\mathbf{R}_{+} ; L^{2}\right)}+\left\|\nabla_{h}\left(u^{\varepsilon}-(\bar{u}, 0)\right)\right\|_{L^{2}\left(\mathbf{R}_{+} ; L^{2}\right)} \rightarrow 0 \text { when } \varepsilon \rightarrow 0 .
$$

Here we shall prove the following result:

Theorem 2. Let $u_{0}$ be a divergence free vector field in $L^{2}$ such that $u_{0 \mid \partial \Omega}^{3}=0$. Let $u^{\varepsilon}$ be a family of weak solutions of $\left(N S C_{\varepsilon}\right)$ associated with $u_{0}$. Denoting by $\bar{u}$ the global solution of the two-dimensional Navier-Stokes 
equations

$$
\begin{gathered}
\Delta_{t} \bar{u}+\operatorname{div}_{h}(\bar{u} \otimes \bar{u})-\nu \Delta_{h} \bar{u}+\nabla^{h} p+\sqrt{2 \beta} \bar{u}=0 \quad \text { in } \quad \mathcal{D}^{\prime}\left(\mathbf{R}^{+} \times \mathbf{R}^{2}\right), \\
\operatorname{div}_{h} \bar{u}=0, \quad \text { and } \bar{u}_{\mid t=0}=\int_{0}^{1} u_{0}\left(x_{h}, x_{3}\right) \mathrm{d} x_{3}
\end{gathered}
$$

we have

$$
\left\|u^{\varepsilon}-(\bar{u}, 0)\right\|_{L^{\infty}\left(\mathbf{R}_{+} ; L_{\mathrm{loc}}^{2}\left(\mathbf{R}^{2} \times\right] 0,1[)\right)}+\left\|\nabla^{h}\left(u^{\varepsilon}-(\bar{u}, 0)\right)\right\|_{L^{2}\left(\mathbf{R}_{+} ; L_{\mathrm{loc}}^{2}\left(\mathbf{R}^{2} \times\right] 0,1[)\right)} \rightarrow 0 \quad \text { when } \varepsilon \rightarrow 0 .
$$

This result is specific to the domain $\left.\mathbf{R}^{2} \times\right] 0,1[$. The key point for the proof of this theorem is that the dispersive phenomenon studied in [4] and [3] is not affected by boundary layers. Let us notice that this result is also true when the family $\left(u^{\varepsilon}\right)$ is associated with a family of initial data $u_{0}^{\varepsilon}$ which converges to some $u_{0}$ strongly in $L^{2}$. The case when the domain is $\left.\mathbf{T}^{2} \times\right] 0,1[$ has been investigated in [10].

To conclude this introduction, let us state some definitions and notations. As the phenomenon studied here is obviously anisotropic, it is natural to introduce the spaces $H^{s, 0}$ which are defined as the closure of smooth compactly supported functions on $\Omega$ for the (semi) norm

$$
\|f\|_{H^{s, 0}} \stackrel{\text { def }}{=}\left(\int_{\left.\mathbf{R}^{2} \times\right] 0,1[}\left|\mathcal{F} f\left(\xi_{h}, x_{3}\right)\right|^{2} \mathrm{~d} \xi_{h} \mathrm{~d} x_{3}\right)^{1 / 2} .
$$

In this spirit we shall introduce

$$
\begin{aligned}
& E_{T}(v) \stackrel{\text { def }}{=} \sup _{0 \leq t \leq T}\left\{\|v(t)\|_{L^{2}}^{2}+\int_{0}^{t}\left\|\nabla^{h} v\left(t^{\prime}\right)\right\|_{L^{2}}^{2} \mathrm{~d} t^{\prime}\right\} \text { and } \\
& \bar{E}_{T}(v) \stackrel{\text { def }}{=} \sup _{0 \leq t \leq T}\left\{\|v(t)\|_{L^{2}}^{2}+\int_{0}^{t}\left\|\nabla^{h} v\left(t^{\prime}\right)\right\|_{L^{2}}^{2} \mathrm{~d} t^{\prime}+\int_{0}^{t}\left\|v\left(t^{\prime}\right)\right\|_{L^{2}}^{2} \mathrm{~d} t^{\prime}\right\} .
\end{aligned}
$$

\section{THE LINEAR PROBLEM IN THE WELL PREPARED CASE}

The goal of this section is to recall some results and methods of [9]. The purpose is to have information on approximate solutions of

$$
\left(F R F^{\varepsilon}\right)\left\{\begin{aligned}
\partial_{t} v^{\varepsilon}-\nu \Delta_{h} v^{\varepsilon}-\beta \varepsilon \partial_{3}^{2} v^{\varepsilon}+\frac{e_{3} \times v^{\varepsilon}}{\varepsilon} & =-\nabla p^{\varepsilon}+f^{\varepsilon} \quad \text { in } \quad \mathbf{R}^{+} \times \Omega \\
\operatorname{div} v^{\varepsilon} & =0 \quad \text { in } \quad \mathbf{R}^{+} \times \Omega \\
v_{\mid t=0}^{\varepsilon} & =v_{0}^{\varepsilon} \quad \text { with } v_{0}^{\varepsilon, 3}=0 \\
v_{\mid \partial \Omega}^{\varepsilon} & =0
\end{aligned}\right.
$$

with

$$
\lim _{\varepsilon \rightarrow 0} f^{\varepsilon}=\bar{f} \quad \text { in } \quad L^{2}\left(\mathbf{R}_{+} ; H^{-1,0}\right) \quad \text { and } \quad \lim _{\varepsilon \rightarrow 0} v_{0}^{\varepsilon}=\bar{v}_{0} \quad \text { in } \quad L^{2}
$$

where $\bar{f}$ belongs to $L^{2}\left(\mathbf{R}^{+} ; H^{-1}\left(\mathbf{R}^{2}\right)\right)$. In all that follows, we shall denote

$$
L^{\varepsilon} v \stackrel{\text { def }}{=} \partial_{t} v-\nu \Delta_{h} v-\beta \varepsilon \partial_{3}^{2} v+\frac{e_{3} \times v}{\varepsilon} .
$$


In [9], it is proved in particular that

$$
\lim _{\varepsilon \rightarrow 0} v^{\varepsilon}=(\bar{v}, 0) \quad \text { in } \quad L^{\infty}\left(\mathbf{R}^{+} ; L^{2}\right) \cap L^{2}\left(\mathbf{R}^{+} ; H^{1,0}\right),
$$

where $\bar{v}$ is the solution of

$$
(L E)\left\{\begin{aligned}
\partial_{t} \bar{v}-\nu \Delta_{h} \bar{v}+\sqrt{2 \beta} \bar{v} & =-\nabla^{h} \bar{p}+\bar{f} \text { in } \quad \mathbf{R}^{+} \times \mathbf{R}^{2} \\
\operatorname{div}_{h} \bar{v} & =0 \quad \text { in } \quad \mathbf{R}^{+} \times \mathbf{R}^{2} \\
\bar{v}_{\mid t=0} & =\bar{v}_{0} \quad \text { with } \quad \bar{v}_{0}^{3}=0 .
\end{aligned}\right.
$$

To simplify notations, we shall note in the following $\bar{v}=(\bar{v}, 0)$.

In fact, much more is done in [9]. We can sum up those results in the following lemma:

Lemma 1. Let $T$ be in $\overline{\mathbf{R}}^{+}$and let $\bar{v}$ be a time dependent divergence free vector field in the space $L^{\infty}([0, T]$; $\left.L^{2}\left(\mathbf{R}^{2}\right)\right)$ whose gradient belongs to $L^{2}\left([0, T] \times \mathbf{R}^{2}\right)$. Let us assume that its Fourier transform is supported in the ball of center 0 and radius $N$. Then a family of smooth divergence free vector fields $\left(v_{\mathrm{app}}^{\varepsilon}\right)_{\varepsilon>0}$ whose value is 0 on the boundary of $\Omega$ exists such that

$$
L^{\varepsilon} v_{\text {app }}^{\varepsilon}=\partial_{t} \bar{v}-\nu \Delta_{h} \bar{v}+\sqrt{2 \beta} \bar{v}+O_{N}\left(\varepsilon^{\frac{1}{2}}\right) \quad \text { in } \quad L^{2}\left([0, T] ; H^{-1,0}\right) .
$$

The vector field $v_{\mathrm{app}}^{\varepsilon}$ tends to $\bar{v}$ in the following sense: a constant $C_{N}$ exists such that

$$
E_{T}\left(v_{\mathrm{app}}^{\varepsilon}-\bar{v}\right) \leq C_{N} \varepsilon^{\frac{1}{2}} E_{T}(\bar{v}) .
$$

Moreover the family $\left(v_{\mathrm{app}}^{\varepsilon}\right)$ satisfies the following estimates

$$
\begin{array}{r}
\int_{0}^{T} \sup _{\left.x_{3} \in\right] 0,1[}\left\|\nabla^{h} v_{\mathrm{app}}^{\varepsilon}\left(t, \cdot, x_{3}\right)\right\|_{L^{2}\left(\mathbf{R}^{2}\right)}^{2} \mathrm{~d} t \leq C_{N} E_{T}(\bar{v}) \\
\forall p \in[2, \infty], \int_{0}^{T} \int_{0}^{1} d\left(x_{3}\right)\left\|\partial_{3} v_{\mathrm{app}}^{\varepsilon}\left(t, \cdot, x_{3}\right)\right\|_{L^{p}\left(\mathbf{R}^{2}\right)}^{2} \mathrm{~d} t \mathrm{~d} x_{3} \leq C_{N} \bar{E}_{T}(\bar{v}) \quad \text { and } \\
\int_{0}^{T} \int_{0}^{1} d\left(x_{3}\right)^{2}\left\|\partial_{3} v_{\mathrm{app}}^{\varepsilon}\left(t, \cdot, x_{3}\right)\right\|_{L^{\infty}\left(\mathbf{R}^{2}\right)}^{2} \mathrm{~d} t \mathrm{~d} x_{3} \leq C_{N} \varepsilon \bar{E}_{T}(\bar{v})
\end{array}
$$

where $d\left(x_{3}\right)$ denotes the distance from $x_{3}$ to the boundary of $] 0,1[$.

For the reader's convenience, let us recall briefly the proof of this lemma. The approximate solution is defined by

$$
\begin{aligned}
v_{\text {app }}^{\varepsilon} \stackrel{\text { def }}{=} & (\bar{v}, 0)-\varepsilon \sqrt{2 \beta}\left(R \bar{v},\left(\frac{1}{2}-x_{3}\right) \bar{\omega}\right)+\left(\left(M_{0}\left(\frac{x_{3}}{\varepsilon \sqrt{2 \beta}}\right)+M_{0}\left(\frac{1-x_{3}}{\varepsilon \sqrt{2 \beta}}\right)-M_{0}\left(\frac{1}{\varepsilon \sqrt{2 \beta}}\right)\right) \bar{v}, 0\right) \\
& +\varepsilon \sqrt{2 \beta}\left(\left(\exp \left(-\frac{x_{3}}{\varepsilon \sqrt{2 \beta}}\right)+\exp \left(-\frac{1-x_{3}}{\varepsilon \sqrt{2 \beta}}\right)-\exp \left(-\frac{1}{\varepsilon \sqrt{2 \beta}}\right)\right) R \bar{v}\right. \\
& \left.\left(f\left(\frac{x_{3}}{\varepsilon \sqrt{2 \beta}}\right)-f\left(\frac{1-x_{3}}{\varepsilon \sqrt{2 \beta}}\right)-\left(1-2 x_{3}\right) f\left(\frac{1}{\varepsilon \sqrt{2 \beta}}\right)\right) \bar{\omega}\right) \\
& +2 \beta \varepsilon^{2}\left(0,\left(\exp \left(-\frac{x_{3}}{\varepsilon \sqrt{2 \beta}}\right)-\exp \left(-\frac{1-x_{3}}{\varepsilon \sqrt{2 \beta}}\right)-\left(1-2 x_{3}\right) \exp \left(-\frac{1}{\varepsilon \sqrt{2 \beta}}\right)\right) \bar{\omega}\right) \\
& +e(\varepsilon \sqrt{2 \beta})\left(-6 x_{3}\left(x_{3}-1\right) R \bar{v}, x_{3}\left(x_{3}-1\right)\left(2 x_{3}-1\right) \bar{\omega}\right)
\end{aligned}
$$


with

$$
M_{0}(\zeta) \stackrel{\text { def }}{=} M_{0}(\zeta)=-e^{-\zeta} R_{-\zeta}, \quad f(\zeta) \stackrel{\text { def }}{=}-\frac{1}{2} e^{-\zeta}(\sin \zeta+\cos \zeta) \quad \text { and } \quad \bar{\omega} \stackrel{\text { def }}{=} \operatorname{curl}_{\mathrm{h}} \overline{\mathrm{v}} .
$$

The function $e(\varepsilon \sqrt{2 \beta})$ is exponentially decreasing and can be computed explicitly. From this formula, the proof of estimates $(1.2,1.3)$ is straightforward.

\section{The LINEAR PROBLEM IN THE ILL PREPARED CASE}

This case is more delicate. The reason why is the following. There is no hope that the term $v_{0, \text { int }}$ does not depend on the vertical variable $x_{3}$, so we have to deal with very fast time oscillations of the interior solution. This will lead us to consider the equation in a different way. The goal of this section is to construct approximate solutions to

$$
\left(F R F^{\varepsilon}\right)\left\{\begin{aligned}
\partial_{t} v^{\varepsilon}-\nu \Delta_{h} v^{\varepsilon}-\beta \varepsilon \partial_{3}^{2} v^{\varepsilon}+\frac{v^{\varepsilon} \times e_{3}}{\varepsilon} & =-\nabla p^{\varepsilon} \quad \text { in } \Omega \\
\operatorname{div} v^{\varepsilon} & =0 \quad \text { in } \quad \Omega \\
v_{\mid t=0}^{\varepsilon} & =v_{0} \\
v_{\mid \partial \Omega}^{\varepsilon} & =0 .
\end{aligned}\right.
$$

Without loss of generality, we can suppose that $v_{0} \in L^{1}(\Omega)$; this will be useful in the proof of Strichartz estimates in Section 4.

First of all, we shall rewrite the system $\left(F R F^{\varepsilon}\right)$ in terms of the Fourier transform of the horizontal divergence and vorticity. To do so, let us decompose the horizontal part of the initial data on the Hilbert basis $\left(\cos \left(k \pi x_{3}\right)\right)_{k \in \mathbf{N}}$ of $L^{2}(] 0,1[)$. We can write for any horizontal vector field $v^{h}$,

$$
v^{h}\left(x_{h}, x_{3}\right)=\sum_{k \in \mathbf{N}} v^{k, h}\left(x_{h}\right) \cos \left(k \pi x_{3}\right)
$$

Note that the fact that $v$ is divergence free implies that

$$
v_{0}^{3}\left(x_{h}, x_{3}\right)=-\sum_{k \geq 1} \frac{1}{k \pi} \operatorname{div}_{h} v_{0}^{k, h}\left(x_{h}\right) \sin \left(k \pi x_{3}\right)
$$

Let us make some preliminary remarks. As the set of functions whose Fourier transform (on $\mathbf{R}^{2}$ ) is supported in rings is dense in $L^{2}$, we can assume that the Fourier transform of the initial data is of this type. Moreover for $L^{2}$ functions, boundary values do not make any sense. However, as $v$ is divergence free, we have $v^{3} \in$ $C\left([0,1] ; H^{-1}\left(\mathbf{R}^{2}\right)\right)$, so the quantity $v_{\mid \partial \Omega}^{3}$ has a meaning.

The case when $k=0$ corresponds to well-prepared case and has been recalled in the previous section. The choice of the basis $\left(\cos \left(k \pi x_{3}\right)\right)_{k \in \mathbf{N}}$ for the horizontal component ensures that the boundary condition $v^{3}{ }_{\mid \partial \Omega}=0$ is satisfied since the vertical component is a linear combination of $\left(\sin \left(k \pi x_{3}\right)\right)_{k \in \mathbf{N}}$ thanks to the divergence free condition. But the terms of type $\cos \left(k \pi x_{3}\right)$ will produce time oscillations. As we shall see later on, the situation is therefore a little bit more complicated than in the well prepared case.

For reasons which will appear clearly when we deal with dispersive phenomena, we need to avoid extreme horizontal frequencies. So we approximate any divergence free vector field $v$ (resp. time dependent) of $L^{2}$ (resp. of $\left.L^{\infty}\left([0, T] ; L^{2}\right) \cap L^{2}\left([0, T] ; H^{1,0}\right)\right)$ by

$$
v_{N} \stackrel{\text { def }}{=} \mathcal{F}^{-1} \sum_{k=0}^{N}\left(\mathbf{1}_{\mathcal{C}_{N}}\left(\xi_{h}\right) \widehat{v}^{k, h}\left(\xi_{h}\right) \cos \left(k \pi x_{3}\right),-\frac{1}{k \pi} \mathbf{1}_{\mathcal{C}_{N}}\left(\xi_{h}\right) \mathcal{F} \operatorname{div}_{h} v_{0}^{k, h}\left(\xi_{h}\right) \sin \left(k \pi x_{3}\right)\right)
$$


where $\mathcal{C}_{N}$ denotes the set of all $\xi_{h}$ in $\mathbf{R}^{2}$ such that $\left|\xi_{h}\right| \in\left[N^{-1}, N\right]$. It is obvious that $\lim _{N \rightarrow \infty} v_{N}=v_{0}$ in $L^{2}$ (resp. in $\left.L^{2}\left([0, T] ; H^{1,0}\right)\right)$.

It is in that situation of boundary conditions that the Rossby operator has a good spectral representation; let us be more precise on that point, before stating the key lemma.

The first step of the construction of the approximate solution consists in writing the system $\left(F R F^{\varepsilon}\right)$ in a simpler way, in fact in terms of the horizontal divergence and horizontal curl of $v^{h}$. Let us state the following notation:

$$
\widehat{d}^{h} \stackrel{\text { def }}{=} \mathcal{F} \operatorname{div}_{h} v^{h}, \quad \widehat{\omega}^{h} \stackrel{\text { def }}{=} \mathcal{F} \operatorname{curl}_{\mathrm{h}} \mathrm{v}^{\mathrm{h}}, \quad \widehat{\mathrm{p}} \stackrel{\text { def }}{=} \mathcal{F} \mathrm{p} \quad \text { and } \quad \mathrm{W} \stackrel{\text { def }}{=}\left(\widehat{\mathrm{d}}^{\mathrm{h}}, \widehat{\omega}^{\mathrm{h}}, \mathcal{F}^{3}\right)
$$

For the sake of simplicity in the notation, we drop the $\varepsilon$ in $\widehat{d}^{h}, \widehat{\omega}^{h}, \widehat{p}^{h}$ and $W$. In the following $\Pi_{1}$ denotes the projection on the first coordinate (recall that the first coordinate of $W$ is the horizontal Fourier transform of the horizontal divergence of the vector field $v$ ). Then for vectors of the type

$$
\left(W^{k, h}(t) \cos \left(k \pi x_{3}\right),-\frac{1}{k \pi} \Pi_{1} W^{k}(t) \sin \left(k \pi x_{3}\right)\right)
$$

writing $W^{k, h}=\left(W^{k, 1}, W^{k, 2}\right)$, and pressures of the type $\widehat{p}^{k} \cos \left(k \pi x_{3}\right)$ the rotating fluid system is equivalent to the following ordinary differential system

$$
\left\{\begin{aligned}
\frac{\mathrm{d}}{\mathrm{d} t} W^{k, 1}+\nu\left|\xi_{h}\right|^{2} W^{k, 1}+\beta \varepsilon(k \pi)^{2} W^{k, 1}-\frac{1}{\varepsilon} W^{k, 2} & =\left|\xi_{h}\right|^{2} \widehat{p}^{k} \\
\frac{\mathrm{d}}{\mathrm{d} t} W^{k, 2}+\nu\left|\xi_{h}\right|^{2} W^{k, 2}+\beta \varepsilon(k \pi)^{2} W^{k, 2}+\frac{1}{\varepsilon} W^{k, 1} & =0 \\
\frac{\mathrm{d}}{\mathrm{d} t} W^{k, 3}+\nu\left|\xi_{h}\right|^{2} W^{k, 3}+\beta \varepsilon(k \pi)^{2} W^{k, 3} & =k \pi \widehat{p}^{k} \\
W^{k, 1}+k \pi W^{k, 3} & =0 \\
W_{\mid t=0} & =W_{0} .
\end{aligned}\right.
$$

As usual the divergence free condition (which here turns out to be $W^{k, 1}+k \pi W^{k, 3}=0$ ) determines the pressure. Here this gives

$$
\widehat{p}^{k}=-\frac{1}{\varepsilon\left(\left|\xi_{h}\right|^{2}+(k \pi)^{2}\right)} W^{k, 2}
$$

The rotating fluid system can now be written

$$
\left(F R F_{k}^{\varepsilon}\right)\left\{\begin{aligned}
\frac{\mathrm{d}}{\mathrm{d} t} W^{k, h}+\nu\left|\xi_{h}\right|^{2} W^{k, h}+\beta \varepsilon(k \pi)^{2} W^{k, h}-\frac{1}{\varepsilon} R_{k} W^{k, h} & =0 \\
W^{k, h}{ }_{\mid t=0} & =W_{0}^{k, h}
\end{aligned}\right.
$$

with

$$
R_{k}=\left(\begin{array}{cc}
0 & -\lambda_{k}^{2} \\
1 & 0
\end{array}\right) \quad \text { and } \quad \lambda_{k} \stackrel{\text { def }}{=}\left(\frac{(k \pi)^{2}}{\left|\xi_{h}\right|^{2}+(k \pi)^{2}}\right)^{\frac{1}{2}}
$$

Now in order to find the interior solution at order zero, and to get rid of the $\varepsilon^{-1}$ terms in the equation, let us 
write

$$
W=\sum_{k=1}^{N}\left(\mathcal{L}_{k}\left(\frac{t}{\varepsilon}\right) \widetilde{W}^{k}(t) \cos \left(k \pi x_{3}\right),-\frac{1}{k \pi} \Pi_{1} \mathcal{L}_{k}\left(\frac{t}{\varepsilon}\right) \widetilde{W}^{k}(t) \sin \left(k \pi x_{3}\right)\right)
$$

where $\mathcal{L}_{k}$ is a function from $\mathbf{R}^{+}$into $\mathcal{L}\left(\mathbf{R}^{2}\right)$ and $\widetilde{W}^{k}$ is a function from $\mathbf{R}^{+}$into $\mathbf{C}^{2}$, to be determined.

Then looking at the terms of size $\varepsilon^{-1}$ in the above equation $\left(F R F_{k}^{\varepsilon}\right)$, we get

$$
\dot{\mathcal{L}}_{k}+R_{k} \mathcal{L}_{k}=0 \quad \text { with } \quad \mathcal{L}_{k}(0)=\mathrm{Id},
$$

which gives

$$
\mathcal{L}_{k}(\tau)=\left(\begin{array}{cc}
\cos \tau_{k} & \lambda_{k} \sin \tau_{k} \\
-\frac{1}{\lambda_{k}} \sin \tau_{k} & \cos \tau_{k}
\end{array}\right)
$$

with, as in all that follows, $\tau_{k}=\lambda_{k} \tau$. Let us remark that when $k=0$ (this corresponds of course to the well prepared case studied in the previous section), we get $\mathcal{L}_{k}=\operatorname{Id}$ because in this case $\lambda_{k}=0$.

To state the approximation lemma in the general case, we need to define the following family of operators $(\mathcal{L}(\tau))_{\tau \in \mathbf{R}}$ : for any vector $v$ of the form $(2.1)$ and $(2.2)$,

$$
\begin{aligned}
(\mathcal{L}(\tau) v)\left(x_{h}, x_{3}\right) \stackrel{\text { def }}{=}\left(v^{0, h}\left(x_{h}\right), 0\right)+\mathcal{F}^{-1} \sum_{k=1}^{\infty}\left(A\left(\xi_{h}\right) \mathcal{L}_{k}(\tau) A^{-1}\left(\xi_{h}\right) \widehat{v}^{k, h}\left(\xi_{h}\right) \cos \left(k \pi x_{3}\right),\right. & \\
& \left.\frac{i}{k \pi} \xi_{h} \cdot A\left(\xi_{h}\right) \mathcal{L}_{k}(\tau) A^{-1}\left(\xi_{h}\right) \widehat{v}^{k, h}\left(\xi_{h}\right) \sin \left(k \pi x_{3}\right)\right)
\end{aligned}
$$

where $\mathcal{L}_{k}$ is defined by formula $(2.3)$ and with

$$
A\left(\xi_{h}\right) \stackrel{\text { def }}{=}\left(\begin{array}{cc}
\xi_{1}\left|\xi_{h}\right|^{-2} & -\xi_{2}\left|\xi_{h}\right|^{-2} \\
\xi_{2}\left|\xi_{h}\right|^{-2} & \xi_{1}\left|\xi_{h}\right|^{-2}
\end{array}\right) .
$$

Let us note that $(\mathcal{L}(\tau))_{\tau \in \mathbf{R}}$ is bounded in $\mathcal{L}\left(H^{s, 0}\right)$ for any real number $s$. Moreover, the restriction of $\mathcal{L}(\tau)$ to functions which do not depend on the third variable (which corresponds to the well prepared case) is Id. This operator $\mathcal{L}(\tau)$ is the Rossby wave operator.

Similarly we shall need the definition of the following "Ekman operator":

$$
\begin{aligned}
&(\mathcal{E} v)\left(x_{h}, x_{3}\right) \stackrel{\text { def }}{=} \sqrt{2 \beta}\left(v^{0, h}\left(x_{h}\right), 0\right)+\mathcal{F}^{-1} \sum_{k=1}^{\infty}\left(A\left(\xi_{h}\right) B_{k} A^{-1}\left(\xi_{h}\right) \widehat{v}^{k, h}\left(\xi_{h}\right) \cos \left(k \pi x_{3}\right),\right. \\
&\left.\frac{i}{k \pi} \xi_{h} \cdot A\left(\xi_{h}\right) B_{k} A^{-1}\left(\xi_{h}\right) \widehat{v}^{k, h}\left(\xi_{h}\right) \sin k \pi x_{3}\right)
\end{aligned}
$$

with

$$
\begin{aligned}
& B_{k} \stackrel{\text { def }}{=} \frac{\left(1-\lambda_{k}^{2}\right) \lambda_{k}}{4}\left(\begin{array}{cc}
\gamma_{k}^{-}-\gamma_{k}^{+} & -\lambda_{k}\left(\gamma_{k}^{+}+\gamma_{k}^{-}\right) \\
\frac{\gamma_{k}^{+}+\gamma_{k}^{-}}{\lambda_{k}} & \gamma_{k}^{-}-\gamma_{k}^{+}
\end{array}\right) \text {and with } \\
& \gamma_{k}^{ \pm} \stackrel{\text { def }}{=}\left(1 \mp \frac{1}{\lambda_{k}}\right) \sqrt{\frac{2 \beta}{1 \pm \lambda_{k}}} .
\end{aligned}
$$


Let us notice that the restriction of $\mathcal{E}$ on functions which do not depend on the third variable (which again corresponds to the well prepared case) is $\sqrt{2 \beta} \mathrm{Id}$. The key lemma is the following:

Lemma 2. Let $\left(v_{N}\right)_{N \in \mathbf{N}}$ be a (time-dependent) bounded sequence of divergence free vector fields in the space

$$
L^{\infty}\left([0, T] ; L^{2}\right) \cap L^{2}\left([0, T] ; H^{1,0}\right)
$$

of the form

$$
v_{N} \stackrel{\text { def }}{=} \mathcal{F}^{-1} \sum_{k=0}^{N}\left(\widehat{v}_{N}^{k, h}\left(\xi_{h}\right) \cos \left(k \pi x_{3}\right),-\frac{1}{i k \pi} \xi_{h} \cdot \widehat{v}_{N}^{k, h}\left(\xi_{h}\right) \sin \left(k \pi x_{3}\right)\right)
$$

with Supp $\widehat{v}_{N}^{\varepsilon, k, h}\left(\xi_{h}\right) \subset \mathcal{C}_{N}$. A sequence of families $\left(v_{\mathrm{app}, N}^{\varepsilon}\right)_{N \in \mathbf{N}}$ of smooth divergence free vector fields whose value on the boundary of $\Omega$ is 0 exists, such that

$$
L^{\varepsilon} v_{\text {app }, N}^{\varepsilon}=\mathcal{L}\left(\frac{t}{\varepsilon}\right)\left(\partial_{t} v_{N}-\nu \Delta_{h} v_{N}+\mathcal{E} v_{N}\right)-\nabla p_{\text {app }, N}^{\varepsilon}+R_{N}^{\varepsilon}
$$

where $R_{N}^{\varepsilon}$ tends to 0 in the following sense:

$$
\forall \eta, \exists N_{1}, \forall N>N_{1}, \exists \varepsilon_{0} / \forall \varepsilon<\varepsilon_{0},\left\|R_{N}^{\varepsilon}\right\|_{L^{\infty}\left(\mathbf{R}^{+} ; H^{-1,0}\right) \cap L^{\infty}\left(\mathbf{R}^{+} ; L^{2}\right)}<\eta .
$$

The vector field $v_{\mathrm{app}, N}^{\varepsilon}$ converges to $\mathcal{L}\left(\frac{t}{\varepsilon}\right) v_{N}$ in the following sense: a constant $C_{N}$ exists such that

$$
E_{T}\left(v_{\mathrm{app}, N}^{\varepsilon}-\mathcal{L}\left(\frac{t}{\varepsilon}\right) v_{N}\right) \leq C_{N} \varepsilon E_{T}\left(v_{N}\right) .
$$

Moreover the family $\left(v_{\mathrm{app}, N}^{\varepsilon}\right)$ satisfies the following estimates

$$
\begin{gathered}
\int_{0}^{T} \sup _{\left.x_{3} \in\right] 0,1[}\left\|\nabla^{h} v_{\mathrm{app}, N}^{\varepsilon}\left(t, \cdot, x_{3}\right)\right\|_{L^{2}\left(\mathbf{R}^{2}\right)}^{2} \mathrm{~d} t \leq C_{N} E_{T}\left(v_{N}\right) \\
\forall p \in[2, \infty], \int_{0}^{T} \int_{0}^{1} d\left(x_{3}\right)\left\|\partial_{3} v_{\mathrm{app}, N}^{\varepsilon}\left(t, \cdot, x_{3}\right)\right\|_{L^{p}\left(\mathbf{R}^{2}\right)}^{2} \mathrm{~d} t \mathrm{~d} x_{3} \leq C_{N} \bar{E}_{T}\left(v_{N}\right) \quad \text { and } \\
\int_{0}^{T} \int_{0}^{1} d\left(x_{3}\right)^{2}\left\|\partial_{3}\left(v_{\mathrm{app}, N}^{\varepsilon}-\mathcal{L}\left(\frac{t}{\varepsilon}\right) v_{N}\right)\right\|_{L^{\infty}\left(\mathbf{R}^{2}\right)}^{2} \mathrm{~d} t \mathrm{~d} x_{3} \leq C_{N} \varepsilon \bar{E}_{T}\left(v_{N}\right) .
\end{gathered}
$$

Remark. In the proof, we shall forget the part associated with $\widehat{v}_{N}^{0, h}$ because this case is nothing but the previous lemma (i.e. the "well-prepared" case).

The proof of this lemma is one of the key points of the paper. In horizontal divergence and curl formulation, the system $\left(F R F^{\varepsilon}\right)$ becomes

$$
\left(\widetilde{F R F} \varepsilon^{\varepsilon}\right)\left\{\begin{aligned}
\partial_{t} W^{1}+\nu\left|\xi_{h}\right|^{2} W^{1}-\beta \varepsilon \partial_{3}^{2} W^{1}-\frac{1}{\xi} W^{2} & =\left|\xi_{h}\right|^{2} \widehat{p} \\
\partial_{t} W^{2}+\nu\left|\xi_{h}\right|^{2} W^{2}-\beta \varepsilon \partial_{3}^{2} W^{2}+\frac{1}{\varepsilon} W^{1} & =0 \\
\partial_{t} W^{3}+\nu\left|\xi_{h}\right|^{2} W^{3}-\beta \varepsilon \partial_{3}^{2} W^{3} & =-\partial_{3} \widehat{p} \\
W^{1}+\partial_{3} W^{3} & =0 \\
W_{\mid t=0} & =W_{0} \\
W_{\mid \partial \Omega} & =0 .
\end{aligned}\right.
$$


From now on, we shall only consider the above system. Let us search for an approximate solution of the form

$$
\begin{aligned}
W & =W_{0, \text { int }}+W_{0, B L}+\varepsilon W_{1, \text { int }}+\varepsilon W_{1, B L}+\cdots \quad \text { and } \\
\widehat{p} & =\frac{1}{\varepsilon} \widehat{p}_{-1, \text { int }}+\frac{1}{\varepsilon} \widehat{p}_{-1, B L}+\widehat{p}_{0, \text { int }}+\widehat{p}_{0, B L}+\cdots
\end{aligned}
$$

where each component of $\left(v_{j, \text { int }}, p_{j, \text { int }}\right)$ is a function of the form

$$
f\left(\frac{t}{\varepsilon}, t, x_{3}\right)
$$

and each component of $\left(v_{j, B L}, p_{j, B L}\right)$ is a function of the form

$$
g\left(\frac{t}{\varepsilon}, t, \frac{x_{3}}{\varepsilon}\right)+h\left(\frac{t}{\varepsilon}, t, \frac{1-x_{3}}{\varepsilon}\right) .
$$

In all that follows, we shall denote $\tau=t / \varepsilon$. First we have to determine the form of $W_{0 \text {,int }}$ and $\widehat{p}_{-1 \text {,int }}$. Considering the decomposition of the initial data, we look for $W_{0, \text { int }}$ of the form

$$
\sum_{k=1}^{N}\left(\mathcal{L}_{k}\left(\frac{t}{\varepsilon}\right) \widetilde{W}_{0, \text { int }}^{k}(t) \cos \left(k \pi x_{3}\right),-\frac{1}{k \pi} \Pi_{1} \mathcal{L}_{k}\left(\frac{t}{\varepsilon}\right) \widetilde{W}_{0, \text { int }}^{k}(t) \sin \left(k \pi x_{3}\right)\right)
$$

where $\mathcal{L}_{k}$ is the function from $\mathbf{R}^{+}$into $\mathcal{L}\left(\mathbf{R}^{2}\right)$ defined above in $(2.3), \widetilde{W}_{0, \text { int }}^{k}$ is a function from $\mathbf{R}^{+}$into $\mathbf{C}^{2}$ and recall that $\Pi_{1}$ denotes the projection on the first coordinate. The vector $\widetilde{W}_{0 \text {,int }}^{k}$ must be understood as the image of $W_{0, \text { int }}^{k, h}$ through the "filtering" operator $\mathcal{L}_{k}$.

Now let us write

$$
W_{0, \text { int }, N} \stackrel{\text { def }}{=} \sum_{k=1}^{N}\left(\mathcal{L}_{k}\left(\frac{t}{\varepsilon}\right) \widetilde{W}_{0, \text { int }}^{k} \cos \left(k \pi x_{3}\right),-\frac{1}{k \pi} \Pi^{1} \mathcal{L}_{k}\left(\frac{t}{\varepsilon}\right) \widetilde{W}_{0, \text { int }}^{k} \sin \left(k \pi x_{3}\right)\right) .
$$

We now have to determine the boundary layer of size $\varepsilon^{0}$. As the third component of the interior solution at order 0 is identically 0 , then the vertical component of the boundary layer of size $\varepsilon^{0}$ vanishes. This implies that $\partial_{3} \widehat{p}_{-1, B L}^{k}=0$. We recover the well known fact that the pressure does not vary in the boundary layer.

Now let us study the term of size $\varepsilon^{-1}$ for the horizontal component of the boundary layer. As $\cos (k \pi)=(-1)^{k}$ we look for the boundary layer of the form

$$
W_{0, B L}^{k, h} \stackrel{\text { def }}{=} M_{k}\left(\frac{x_{3}}{\varepsilon}\right) \mathcal{L}_{k}\left(\frac{t}{\varepsilon}\right) \widetilde{W}_{0, \text { int }}^{k}+(-1)^{k} M_{k}\left(\frac{1-x_{3}}{\varepsilon}\right) \mathcal{L}_{k}\left(\frac{t}{\varepsilon}\right) \widetilde{W}_{0, \text { int }}^{k} .
$$

The term of size $\varepsilon^{-1}$ of

$$
\partial_{t} W_{0, B L}^{k, h}-\beta \varepsilon \partial_{3}^{2} W_{0, B L}^{k, h}+\frac{1}{\varepsilon} R W_{0, B L}^{k, h}
$$

must be equal to 0 . So we infer that

$$
M_{k} \dot{\mathcal{L}}_{k}-\beta M_{k}^{\prime \prime} \mathcal{L}_{k}+R M_{k} \mathcal{L}_{k}=0
$$


Let us notice that in the case when $k=0$, we have $\mathcal{L}_{k}=$ Id. Now let us assume that $k \geq 1$. As $\dot{\mathcal{L}}_{k}=-R_{k} \mathcal{L}_{k}$, it turns out that the equation on the boundary layer is

$$
\left\{\begin{aligned}
-\beta M_{k}^{\prime \prime} & =M_{k} R_{k}-R M_{k} \\
M_{k}(0) & =-\mathrm{Id} \\
M_{k}(+\infty) & =0
\end{aligned}\right.
$$

This is a linear differential equation of order 2 with an initial and a final condition. The solution is

$$
M_{k}(\zeta)=-\sum_{ \pm} \frac{1}{2} \mu_{k}^{ \pm} \exp \left(-\zeta_{k}^{ \pm}\right) M_{k}^{ \pm}\left(\zeta_{k}^{ \pm}\right)
$$

with

$$
M_{k}^{ \pm}(\theta) \stackrel{\text { def }}{=}\left(\begin{array}{cc}
\cos \theta & \mp \lambda_{k} \sin \theta \\
-\sin \theta & \mp \lambda_{k} \cos \theta
\end{array}\right)
$$

and

$$
\zeta_{k}^{ \pm} \stackrel{\text { def }}{=} \frac{\zeta}{\sqrt{2 \beta_{k}^{ \pm}}}, \beta_{k}^{ \pm} \stackrel{\text { def }}{=} \frac{\beta}{1 \pm \lambda_{k}} \quad \text { and } \quad \mu_{k}^{ \pm} \stackrel{\text { def }}{=} 1 \mp \frac{1}{\lambda_{k}}
$$

So stating

$$
E_{k}^{ \pm} \stackrel{\text { def }}{=} 2 \varepsilon^{2} \beta_{k}^{ \pm}
$$

we infer by definition of $\mathcal{L}_{k}$ that

$$
\begin{aligned}
W_{0, B L}^{k, h}=-\frac{1}{2} \sum_{ \pm} \mu_{k}^{ \pm} \exp \left(-\frac{x_{3}}{\sqrt{E_{k}^{ \pm}}}\right) M_{k}^{ \pm}\left(\frac{x_{3}}{\sqrt{E_{k}^{ \pm}}} \mp \frac{\lambda_{k} t}{\varepsilon}\right) \widetilde{W}_{0, \text { int }}^{k} \\
\quad-(-1)^{k} \frac{1}{2} \sum_{ \pm} \mu_{k}^{ \pm} \exp \left(-\frac{1-x_{3}}{\sqrt{E_{k}^{ \pm}}}\right) M_{k}^{ \pm}\left(\frac{1-x_{3}}{\sqrt{E_{k}^{ \pm}}} \frac{\lambda_{k} t}{\varepsilon}\right) \widetilde{W}_{0, \text { int }}^{k} .
\end{aligned}
$$

Let us remark that the eigenvectors of $M_{k}^{ \pm}$and those of $\mathcal{L}_{k}$ are different. Moreover, time oscillations introduce a phase shift in the boundary layer.

As in the well prepared case, the fact that the boundary layer must be divergence free implies that we have to introduce a vertical component of the boundary layer of size $\varepsilon$. It is given by the following formula:

$$
\varepsilon \partial_{3} W_{1, B L}^{k, 3}=-\Pi_{1} W_{0, B L}^{k, h}
$$


So we get

$$
\begin{aligned}
\varepsilon \partial_{3} W_{1, B L}^{k, 3}= & \frac{1}{2} \sum_{ \pm} \mu_{k}^{ \pm} \exp \left(-\frac{x_{3}}{\sqrt{E_{k}^{ \pm}}}\right)\left(\cos \left(\frac{x_{3}}{\sqrt{E_{k}^{ \pm}}} \mp \frac{\lambda_{k} t}{\varepsilon}\right) \widetilde{W}_{0, \text { int }}^{k, 1}\right. \\
& \left.\mp \lambda_{k} \sin \left(\frac{x_{3}}{\sqrt{E_{k}^{ \pm}}} \mp \frac{\lambda_{k} t}{\varepsilon}\right) \widetilde{W}_{0, \text { int }}^{k, 2}\right) \\
& +(-1)^{k} \frac{1}{2} \sum_{ \pm} \mu_{k}^{ \pm} \exp \left(-\frac{1-x_{3}}{\sqrt{E_{k}^{ \pm}}}\right)\left(\cos \left(\frac{1-x_{3}}{\sqrt{E_{k}^{ \pm}}} \mp \frac{\lambda_{k} t}{\varepsilon}\right) \widetilde{W}_{0, \text { int }}^{k, 1}\right. \\
& \left.\mp \lambda_{k} \sin \left(\frac{1-x_{3}}{\sqrt{E_{k}^{ \pm}}} \mp \frac{\lambda_{k} t}{\varepsilon}\right) \widetilde{W}_{0, \text { int }}^{k, 2}\right) .
\end{aligned}
$$

So with the notation

$$
\mathrm{cs}^{ \pm} \stackrel{\text { def }}{=} \cos \pm \sin \quad \text { and } \quad \gamma_{k}^{ \pm} \stackrel{\text { def }}{=} \mu_{k}^{ \pm} \sqrt{2 \beta_{k}^{ \pm}}
$$

we get by integration,

$$
\begin{aligned}
W_{1, B L}^{k, 3}= & -\frac{1}{4} \sum_{ \pm} \gamma_{k}^{ \pm} \exp \left(-\frac{x_{3}}{\sqrt{E_{k}^{ \pm}}}\right)\left(\mathrm{cs}^{-}\left(\frac{x_{3}}{\sqrt{E_{k}^{ \pm}}} \mp \frac{\lambda_{k} t}{\varepsilon}\right) \widetilde{W}_{0, \text { int }}^{k, 1}\right. \\
& \left.\mp \lambda_{k} \mathrm{cs}^{+}\left(\frac{x_{3}}{\sqrt{E_{k}^{ \pm}}} \mp \frac{\lambda_{k} t}{\varepsilon}\right) \widetilde{W}_{0, \text { int }}^{k, 2}\right) \\
& +(-1)^{k} \frac{1}{4} \sum_{ \pm} \gamma_{k}^{ \pm} \exp \left(-\frac{1-x_{3}}{\sqrt{E_{k}^{ \pm}}}\right)\left(\operatorname{cs}^{-}\left(\frac{1-x_{3}}{\sqrt{E_{k}^{ \pm}}} \mp \frac{\lambda_{k} t}{\varepsilon}\right) \widetilde{W}_{0, \text { int }}^{k, 1}\right. \\
& \left.\mp \lambda_{k} \mathrm{cs}^{+}\left(\frac{1-x_{3}}{\sqrt{E_{k}^{ \pm}}} \mp \frac{\lambda_{k} t}{\varepsilon}\right) \widetilde{W}_{0, \text { int }}^{k, 2}\right) .
\end{aligned}
$$

It is clear that this boundary layer is a sum of a rapidly decreasing function of $x_{3} / \varepsilon$ and of a rapidly decreasing function of $\left(1-x_{3}\right) / \varepsilon$. But it is obvious that those two functions do not vanish respectively in $x_{3}=0$ and $x_{3}=1$. More precisely, up to exponentially small terms, we have, stating again $\tau_{k}=\lambda_{k} \tau$,

$$
W_{1, B L_{\mid x_{3}=0}}^{k, 3}=-(-1)^{k} W_{1, B L_{\mid x_{3}=1}^{k, 3}}^{k}=-f_{k}\left(\frac{t}{\varepsilon}\right)
$$

with

$$
f_{k}(\tau) \stackrel{\text { def }}{=} \sum_{ \pm} \frac{\gamma_{k}^{ \pm}}{4}\left(\operatorname{cs}^{ \pm}\left(\tau_{k}\right) \widetilde{W}_{0, \text { int }}^{k, 1} \mp \lambda_{k} \operatorname{cs}^{\mp}\left(\tau_{k}\right) \widetilde{W}_{0, \text { int }}^{k, 2}\right)
$$


Now, let us have a look at the terms of size $\varepsilon^{0}$ in the interior system. The system of equations is the following.

$$
\left\{\begin{aligned}
\left(\partial_{t}+\nu\left|\xi_{h}\right|^{2}\right) W_{0, \text { int }, N}^{h}+\partial_{\tau} W_{1, \text { int }, N}^{h}+R W_{1, \text { int }, N}^{h} & =\left(\begin{array}{c}
\left.\xi_{h}\right|^{2} \widehat{p}_{0, \text { int }, N} \\
0
\end{array}\right) \\
\left(\partial_{t}+\nu\left|\xi_{h}\right|^{2}\right) W_{0, \text { int }, N}^{3}+\partial_{\tau} W_{1, \text { int }, N}^{3} & =-\partial_{3} \widehat{p}_{0, \text { int }, N} \\
W_{1, \text { int }, N}^{1}+\partial_{3} W_{1, \text { int }, N}^{3} & =0 \\
W_{1, \text { int }, N \mid \partial \Omega}^{3} & =-W_{1, B L \mid \partial \Omega}^{k, 3} .
\end{aligned}\right.
$$

As usual, we are going to reduce this problem to a problem with a homogeneous Dirichlet boundary condition. To do so, let us first define the function $r_{\ell}$ where

$$
r_{\ell}\left(x_{3}\right)=1 \quad \text { when } \ell \text { is odd, } \quad r_{\ell}\left(x_{3}\right)=1-2 x_{3} \quad \text { when } \ell \text { is even. }
$$

Then let us state

$$
\underline{W}_{1, \text { int }, N} \stackrel{\text { def }}{=} \sum_{\ell=1}^{N} f_{\ell}(\tau)\left(\delta_{\ell}, 0, r_{\ell}\left(x_{3}\right)\right) \quad \text { with } \quad \delta_{\ell}=1+(-1)^{\ell}
$$

and let us look for $W_{1, \text { int }, N}$ of the form

$$
W_{1, \text { int }, N}=\bar{W}_{1, \text { int }, N}+\underline{W}_{1, \text { int }, N} .
$$

Now the above system can be written

$$
\left\{\begin{aligned}
\left(\partial_{t}+\nu\left|\xi_{h}\right|^{2}\right) W_{0, \text { int }, N}^{h}+\left(\partial_{\tau}+R\right) \bar{W}_{1, \text { int }, N}^{h} & =\left(\begin{array}{c}
\left|\xi_{h}\right|^{2} \widehat{p}_{0, \text { int }, N} \\
0
\end{array}\right)-\left(\partial_{\tau}+R\right) \underline{W}_{1, \text { int }, N}^{h} \\
\left(\partial_{t}+\nu\left|\xi_{h}\right|^{2}\right) W_{0, \text { int }, N}^{3}+\partial_{\tau} \bar{W}_{1, \text { int }, N}^{3} & =-\partial_{3} \widehat{p}_{0, \text { int }, N}-\partial_{\tau} \underline{W}_{1, \text { int }, N}^{3} \\
\bar{W}_{1, \text { int }, N}^{1}+\partial_{3} \bar{W}_{1, \text { int }, N}^{3} & =0 \\
\bar{W}_{1, \text { int }, N \mid \partial \Omega}^{3} & =0 .
\end{aligned}\right.
$$

Still as usual, the original boundary condition appears through a forcing term. Considering this boundary condition on $\bar{W}_{1, \text { int, } N}$, it is natural to look for $\bar{W}_{1, \text { int }, N}$ and $\widehat{p}_{0, \text { int }, N}$ of the form

$$
\begin{aligned}
\bar{W}_{1, \text { int }, N} & =\left(\sum_{k=0}^{N} W_{1, \text { in }, N}^{k, h} \cos \left(k \pi x_{3}\right),-\sum_{k=1}^{N} \frac{1}{k \pi} W_{1, \text { int }, N}^{k, 1} \sin \left(k \pi x_{3}\right)\right) \quad \text { and } \\
\widehat{p}_{0, \text { int }, N} & =\sum_{k=0}^{N} p_{0, \text { int }}^{k} \cos \left(k \pi x_{3}\right) .
\end{aligned}
$$

Now let us decompose the forcing term in a low and a high vertical frequency part. This part will be small when $N$ tends to $\infty$. Let us notice that in $L^{2}(] 0,1[)$, we have

$$
r_{\ell}\left(x_{3}\right)=\sum_{k \geq 1} r_{\ell, k} \sin \left(k \pi x_{3}\right) \quad \text { with } \quad r_{\ell, k} \stackrel{\text { def }}{=} \frac{1}{k \pi}\left(1+(-1)^{k+\ell}\right) .
$$

So, we write that

$$
\underline{W}_{1, \mathrm{int}, N}=F_{N}+R_{N}^{\varepsilon} \quad \text { with } \quad F_{N} \stackrel{\text { def }}{=} \sum_{\ell=1}^{N} f_{\ell}(\tau)\left(\delta_{\ell}, 0, \sum_{k=1}^{N} r_{\ell, k} \sin \left(k \pi x_{3}\right)\right) .
$$


Obviously, $R_{N}^{\varepsilon}$ satisfies the property (2.6). Now let us project on cosine and sine functions, which yields

$$
\begin{aligned}
\left(\frac{\mathrm{d}}{\mathrm{d} \tau}+R\right) \bar{W}_{1, \text { int }, N}^{0, h} & =\sum_{\ell=1}^{N}\left\{-\frac{\mathrm{d} f_{\ell}}{\mathrm{d} \tau}\left(\delta_{\ell}, 0\right)+f_{\ell}(\tau)\left(0, \delta_{\ell}\right)\right\} \\
\frac{\mathrm{d}}{\mathrm{d} \tau} \bar{W}_{1, \text { int }, N}^{0,3} & =0
\end{aligned}
$$

and, when $k \neq 0$, considering the form of $W_{0, \text { int }, N}$ given by (2.11)

$$
\begin{aligned}
& \mathcal{L}_{k}(\tau)\left(\frac{\mathrm{d}}{\mathrm{d} t}+\nu\left|\xi_{h}\right|^{2}\right) \widetilde{W}_{0, \text { int }}^{k}+\left(\frac{\mathrm{d}}{\mathrm{d} \tau}+R\right) \bar{W}_{1, \text { int }, N}^{k, h}=\left(\begin{array}{c}
\left|\xi_{h}\right|^{2} \widehat{p}_{0, \text { int }}^{k} \\
0
\end{array}\right) \\
& -\Pi_{1} \frac{1}{k \pi} \mathcal{L}_{k}(\tau)\left(\frac{\mathrm{d}}{\mathrm{d} t}+\nu\left|\xi_{h}\right|^{2}\right) \widetilde{W}_{0, \text { int }}^{k}+\frac{\mathrm{d}}{\mathrm{d} \tau} \bar{W}_{1, \text { int }, N}^{k, 3}=-\sum_{\ell \leq N} r_{\ell, k} \frac{\mathrm{d} f_{\ell}}{\mathrm{d} \tau}+k \pi \widehat{p}_{0, \text { int }}^{k} .
\end{aligned}
$$

Let us solve first the system when $k=0$. As $\bar{W}_{1, \text { int }, N}^{0,3}=0$ the divergence free condition implies that $\bar{W}_{1, \text { int }, N}^{0,1}=0$. So the system becomes

$$
\begin{aligned}
-\bar{W}_{1, \text { int }, N}^{0,2} & =-\sum_{\ell=1}^{N} \frac{\mathrm{d} f_{\ell}}{\mathrm{d} \tau}+\left|\xi_{h}\right|^{2} \widehat{p}_{0, \text { int }, N}^{0} \\
\frac{\mathrm{d}}{\mathrm{d} \tau} \bar{W}_{1, \text { int }, N}^{0,2} & =\sum_{\ell=1}^{N} f_{\ell}(\tau) .
\end{aligned}
$$

Thus we get

$$
W_{1, \text { int }, N}^{0}=\left(0, \sum_{\ell=1}^{N} \sum_{ \pm} \frac{\gamma_{\ell}^{ \pm}}{4 \lambda_{\ell}}\left(\mp \mathrm{cs}^{\mp}\left(\tau_{\ell}\right) \widetilde{W}_{0, \text { int }}^{\ell, 1}+\lambda_{\ell} \operatorname{cs}^{ \pm}\left(\tau_{\ell}\right) \widetilde{W}_{0, \text { int }}^{\ell, 2}\right), 0\right)
$$

We also get an explicit formula for $p_{0, \text { int }, N}^{0}$ whose computation is left to the reader.

Let us notice that those terms do not depend on the variable $x_{3}$ and are bounded in time and time oscillating.

Now let us study the case when $k \neq 0$. As usual, the divergence free condition will determine the pressure by

$$
\widehat{p}_{0, \text { int }}^{k}=\frac{1}{\left|\xi_{h}\right|^{2}+(k \pi)^{2}}\left(\sum_{\ell \leq N} k \pi r_{\ell, k} \frac{\mathrm{d} f_{\ell}}{\mathrm{d} \tau}+\bar{W}_{1, \text { int }, N}^{k, 2}\right) .
$$

So now the system becomes

$$
\mathcal{L}_{k}(\tau)\left(\frac{\mathrm{d}}{\mathrm{d} t}+\nu\left|\xi_{h}\right|^{2}\right) \widetilde{W}_{0, \text { int }}^{k}+\left(\frac{\mathrm{d}}{\mathrm{d} \tau}+R_{k}\right) \bar{W}_{1, \mathrm{int}, N}^{k, h}-\sum_{\ell \leq N}\left(\begin{array}{c}
\frac{k \pi r_{\ell, k}\left|\xi_{h}\right|^{2}}{\left|\xi_{h}\right|^{2}+(k \pi)^{2}} \frac{\mathrm{d} f_{\ell}}{\mathrm{d} \tau} \\
0
\end{array}\right)=0 .
$$

Now we have to remember that the $f_{\ell}$ are linear maps of $\widetilde{W}_{0 \text {,int }}^{\ell}$ whose coefficients oscillate in time with the frequency $\frac{\lambda_{\ell}}{\varepsilon}$. This leads to the following lemma whose proof is a series of straightforward computations left to the reader. 
Lemma 3. We have the following identity:

$$
-\sum_{\ell \leq N}\left(\begin{array}{c}
\frac{k \pi r_{\ell, k}\left|\xi_{h}\right|^{2}}{\left|\xi_{h}\right|^{2}+(k \pi)^{2}} \frac{\mathrm{d} f_{\ell}}{\mathrm{d} \tau} \\
0
\end{array}\right)=\mathcal{L}_{k}(\tau)\left(B_{k}+\sum_{\ell=1}^{N} B_{k, \ell}(\tau)\right) \widetilde{W}_{0, \text { int }}^{k}
$$

where $B_{k, \ell}(\tau)$ are matrices whose coefficients are cosine or sine functions of $\left(\lambda_{k} \pm \lambda_{\ell}\right) \tau$ for $\ell \neq k$ and of $\lambda_{k} \tau$ when $\ell=k$ and where

$$
B_{k}=\frac{\left(1-\lambda_{k}^{2}\right) \lambda_{k}}{4}\left(\begin{array}{cc}
\gamma_{k}^{-}-\gamma_{k}^{+} & -\lambda_{k}\left(\gamma_{k}^{+}+\gamma_{k}^{-}\right) \\
\frac{\gamma_{k}^{+}+\gamma_{k}^{-}}{\lambda_{k}} & \gamma_{k}^{-}-\gamma_{k}^{+}
\end{array}\right)
$$

Thus the system splits into the two following ones

$$
\begin{aligned}
\left(\frac{\mathrm{d}}{\mathrm{d} t}+\nu\left|\xi_{h}\right|^{2}+B_{k}\right) \widetilde{W}_{0, \text { int }}^{k} & =0 \quad \text { and } \\
\left(\frac{\mathrm{d}}{\mathrm{d} \tau}+R_{k}\right) \bar{W}_{1, \text { int }, N}^{k, h} & =-\mathcal{L}_{k}(\tau)\left(\sum_{\ell=1}^{N} B_{k, \ell}(\tau)\right) \widetilde{W}_{0, \text { int }}^{k}
\end{aligned}
$$

Immediately we have that

$$
\begin{aligned}
& \widetilde{W}_{0, \text { int }}^{k}(t)=\exp \left(-\nu\left|\xi_{h}\right|^{2} t-P_{k} t\right)\left(\begin{array}{cc}
\cos \delta_{k} t & \lambda_{k} \sin \delta_{k} t \\
-\frac{1}{\lambda_{k}} \sin \delta_{k} t & \cos \delta_{k} t
\end{array}\right) \widetilde{W}_{0, \text { int }}^{k}(0) \text { with } \\
& \delta_{k} \stackrel{\text { def }}{=} \frac{\left(1-\lambda_{k}^{2}\right) \lambda_{k}}{4}\left(\gamma_{k}^{+}+\gamma_{k}^{-}\right), \\
& P_{k} \stackrel{\text { def }}{=} \frac{\left(1-\lambda_{k}^{2}\right) \lambda_{k}}{4}\left(\gamma_{k}^{-}-\gamma_{k}^{+}\right) \quad \text { and } \\
& W_{1, \text { int }, N}^{k, h}=\mathcal{L}_{k}\left(\frac{t}{\varepsilon}\right) \sum_{\ell=1}^{N} C_{k, \ell}\left(\frac{t}{\varepsilon}\right) \widetilde{W}_{0, \text { int }}^{k}(t)
\end{aligned}
$$

where $C_{k, \ell}$ are $(2 \times 2$ valued $)$ smooth bounded functions of $\tau$ whose derivatives are the $B_{k, \ell}$. So applying the usual procedure for the higher order terms for boundary layers, we find the complete expression of the approximate solution.

As far as estimates (2.9) and (2.10) are concerned, the worse terms are obviously $W_{0, B L}^{k, h}$. As the support of $\mathcal{F} W_{0, B L}^{k, h}$ is included in $\mathcal{C}\left(N^{-1}, N\right)$, it is enough estimate then $L^{2}$ norm on $\mathbf{R}^{2}$.

From the above formula, we have that

$$
\left\|\partial_{3} W_{0, B L}^{k, h}\left(t, \cdot, x_{3}\right)\right\|_{L^{2}\left(\mathbf{R}^{2}\right)} \leq \frac{C_{k}}{\varepsilon} \sum_{ \pm}\left(\exp \left(-\frac{x_{3}}{\varepsilon \sqrt{2 \beta_{k}^{ \pm}}}\right)+\exp \left(-\frac{1-x_{3}}{\varepsilon \sqrt{2 \beta_{k}^{ \pm}}}\right)\right)\left\|\widetilde{W}_{0, \text { int }}^{k, h}(t, \cdot)\right\|_{L^{2}\left(\mathbf{R}^{2}\right)}
$$

So we infer that

$$
\begin{aligned}
\int_{0}^{1} \mathrm{~d}\left(x_{3}\right)\left\|\partial_{3} W_{0, B L}^{k, h}\left(t, \cdot, x_{3}\right)\right\|_{L^{2}\left(\mathbf{R}^{2}\right)}^{2} & \leq C_{k}\left\|W_{0, \text { int }}^{k, h}(t, \cdot)\right\|_{L^{2}\left(\mathbf{R}^{2}\right)}^{2} \int_{0}^{1} \frac{\mathrm{d}\left(x_{3}\right)}{\varepsilon} \exp \left(-2 \frac{\mathrm{d}\left(x_{3}\right)}{\varepsilon}\right) \mathrm{d} x_{3} \\
& \leq C_{k}\left\|W_{0, \text { int }}^{k, h}(t, \cdot)\right\|_{L^{2}\left(\mathbf{R}^{2}\right)}^{2}
\end{aligned}
$$


Moreover, exactly along the same lines, we have that

$$
\begin{aligned}
\int_{0}^{1} \mathrm{~d}\left(x_{3}\right)^{2}\left\|\partial_{3} W_{0, B L}^{k, h}\left(t, \cdot, x_{3}\right)\right\|_{L^{2}\left(\mathbf{R}^{2}\right)} & \leq C_{k}\left\|W_{0, \text { int }}^{k, h}(t, \cdot)\right\|_{L^{2}\left(\mathbf{R}^{2}\right)}^{2} \int_{0}^{1} \frac{\mathrm{d}\left(x_{3}\right)^{2}}{\varepsilon} \exp \left(-2 \frac{\mathrm{d}\left(x_{3}\right)}{\varepsilon}\right) \mathrm{d} x_{3} \\
& \leq C_{k} \varepsilon\left\|W_{0, \text { int }}^{k, h}(t, \cdot)\right\|_{L^{2}\left(\mathbf{R}^{2}\right)}^{2} .
\end{aligned}
$$

The result follows.

\section{THE NON LINEAR ESTIMATES}

This section consists in proving Theorem 2. Let us first cut off the initial data in the frequency space. To do so, let us define

$$
\begin{aligned}
& \bar{u}_{0}\left(x_{h}\right) \stackrel{\text { def }}{=} \int_{0}^{1} u_{0}\left(x_{h}, x_{3}\right) \mathrm{d} x_{3}, \\
& u_{0}^{k, h}\left(x_{h}\right) \stackrel{\text { def }}{=} \frac{1}{2} \int_{0}^{1} u_{0}\left(x_{h}, x_{3}\right) \cos \left(k \pi x_{3}\right) \mathrm{d} x_{3}, \\
& \bar{u}_{0, N} \stackrel{\text { def }}{=} \mathcal{F}^{-1}\left(\mathbf{1}_{\mathcal{C}_{N}} \widehat{\bar{u}}_{0}\right) \text { and } \\
& u_{0, N}^{k, h} \stackrel{\stackrel{\text { def }}{=}}{\mathcal{F}^{-1}\left(\mathbf{1}_{\mathcal{C}_{N}} \widehat{u}_{0}^{k, h}\right) .}
\end{aligned}
$$

For any positive real number $\eta$, an integer $N_{0}$ exists, depending of course on $\eta$ and on the initial data $u_{0}$ such that

$$
\begin{aligned}
\left\|u_{0}-\bar{u}_{0, N}-\widetilde{u}_{0, N}\right\|_{L^{2}} & <\frac{\eta}{4} \quad \text { with } \\
\widetilde{u}_{0, N} & =\sum_{k=1}^{N}\left(u_{0, N}^{k, h}\left(x_{h}\right) \cos \left(k \pi x_{3}\right),-\frac{1}{k \pi} \operatorname{div}_{h} u_{0, N}^{k, h}\left(x_{h}\right) \sin \left(k \pi x_{3}\right)\right) .
\end{aligned}
$$

Let us define $\bar{u}_{N}$ as the solution of

$$
\left(L E_{\nu, \beta}\right)\left\{\begin{aligned}
\Delta_{t} \bar{u}_{N}-\nu \Delta_{h} \bar{u}_{N}+\sqrt{2 \beta} \bar{u}_{N} & =-\nabla \bar{p}_{N}-\mathcal{F}^{-1}\left(\mathbf{1}_{\mathcal{C}_{N}} \mathcal{F}\left(\operatorname{div}_{h}(\bar{u} \otimes \bar{u})\right)\right) \\
\operatorname{div}_{h} \bar{u}_{N} & =0 \\
\bar{u}_{N \mid t=0} & =\bar{u}_{0, N} .
\end{aligned}\right.
$$

A basic energy estimate implies that, for any positive $\eta$, an integer $N_{0}$ exists such that, for any $t$ in $\mathbf{R}^{+}$and any $N$ greater or equal to $N_{0}$, we have

$$
\left\|\bar{u}(t)-\bar{u}_{N}(t)\right\|_{L^{2}}^{2}+2 \int_{0}^{t}\left(\nu\left\|\nabla^{h}\left(\bar{u}\left(t^{\prime}\right)-\bar{u}_{N}\left(t^{\prime}\right)\right)\right\|_{L^{2}}^{2}+\sqrt{2 \beta}\left\|\bar{u}\left(t^{\prime}\right)-\bar{u}_{N}\left(t^{\prime}\right)\right\|_{L^{2}}^{2}\right) \mathrm{d} t^{\prime}<\frac{\eta^{2}}{16} .
$$

Sobolev embeddings also imply that $\bar{u}$ belongs to $L^{4}\left(\mathbf{R}^{+} \times \mathbf{R}^{2}\right)$ and $\bar{u}_{N}$ converges to $\bar{u}$ in the space $L^{4}\left(\mathbf{R}^{+} \times \mathbf{R}^{2}\right)$. Thus we have

$$
\lim _{N \rightarrow \infty} \mathcal{F}^{-1}\left(\mathbf{1}_{\mathcal{C}_{N}} \mathcal{F}\left(\operatorname{div}_{h}(\bar{u} \otimes \bar{u})\right)\right)=\lim _{N \rightarrow \infty} \operatorname{div}_{h}\left(\bar{u}_{N} \otimes \bar{u}_{N}\right)=\operatorname{div}_{h}(\bar{u} \otimes \bar{u})
$$

in the space $L^{2}\left(\mathbf{R}^{+} ; H^{-1}\left(\mathbf{R}^{2}\right)\right)$.

Now we shall use Lemmas 1 and 2 to define the (sequence of) approximate solutions of the system. Let us define $\left(u_{w p, N}^{\varepsilon}\right)_{\varepsilon>0}$ as the families given by Lemma 1 applied with $v_{N}=\bar{u}_{N}$ solution of $\left(L E_{\nu, \beta}\right)$; it represents the 
"well prepared" part of the solution. The so-called "ill prepared" part is defined as $\left(u_{i p, N}^{\varepsilon}\right)$, the families given by Lemma 2 applied with $v_{N}$ equal to the solution of the linear problem

$$
\left\{\begin{aligned}
\partial_{t} v_{N}-\nu \Delta_{h} v_{N}+\mathcal{E} v_{N} & =-\nabla p_{N} \\
\operatorname{div} v_{N} & =0 \\
v_{N \mid t=0} & =\widetilde{u}_{0, N}
\end{aligned}\right.
$$

Of course, we state

$$
u_{\mathrm{app}, N}^{\varepsilon} \stackrel{\text { def }}{=} u_{w p, N}^{\varepsilon}+u_{i p, N}^{\varepsilon} .
$$

Let us derive the equation satisfied by $u_{\mathrm{app}, N}^{\varepsilon}$. Using Lemmas 1, 2 and (3.2), we get that

$$
L^{\varepsilon} u_{\mathrm{app}, N}^{\varepsilon}=R_{N}^{\varepsilon}+\nabla p_{\varepsilon}+\operatorname{div}_{h}\left(\bar{u}_{N} \otimes \bar{u}_{N}\right) .
$$

Using energy estimates, we get that

$$
\begin{aligned}
\left\|u_{\mathrm{app}, N}^{\varepsilon}(t)\right\|_{L^{2}}^{2}+2 \nu \int_{0}^{t}\left\|\nabla^{h} u_{\mathrm{app}, N}^{\varepsilon}\left(t^{\prime}\right)\right\|_{L^{2}}^{2} \mathrm{~d} t^{\prime} & +2 \beta \varepsilon \int_{0}^{t}\left\|\partial_{3} u_{\mathrm{app}, N}^{\varepsilon}\left(t^{\prime}\right)\right\|_{L^{2}}^{2} \mathrm{~d} t^{\prime} \\
& \leq\left\|u_{0}\right\|_{L^{2}}^{2}-\int_{0}^{t}\left(\operatorname{div}_{h}\left(\bar{u}_{N} \otimes \bar{u}_{N}\right)\left(t^{\prime}\right) \mid u_{\mathrm{app}, N}^{\varepsilon}\left(t^{\prime}\right)\right)_{L^{2}} \mathrm{~d} t^{\prime}+\rho_{N}^{\varepsilon}
\end{aligned}
$$

where, as in all that follows, $\rho_{N}^{\varepsilon}$ denotes generically a scalar quantity such that

$$
\forall \eta, \exists N_{0}, \forall N>N_{0}, \exists \varepsilon_{0} / \forall \varepsilon<\varepsilon_{0}, \rho_{N}^{\varepsilon}<\eta .
$$

In fact, $\rho_{N}^{\varepsilon}$ will be the sum of a series which tends to 0 uniformly in $\varepsilon$ when $N$ goes to $\infty$ and of terms bounded by $C_{N} \varepsilon^{\alpha}$ for some positive $\alpha$. Thanks to Inequality (2.7) of Lemma 2 we have

$$
\left\|u_{\mathrm{app}, N}^{\varepsilon}-\mathcal{L}\left(\frac{t}{\varepsilon}\right) v_{N}\right\|_{L^{2}\left(\mathbf{R}^{+} ; H^{1,0}\right)} \leq C_{N} \varepsilon^{\frac{1}{2}}\left\|u_{0}\right\|_{L^{2}} .
$$

So, as $\operatorname{div}_{h}\left(\bar{u}_{N} \otimes \bar{u}_{N}\right)$ belongs to $L^{2}\left(\mathbf{R}^{+} \times \Omega\right)$, we have

$$
\begin{aligned}
\int_{0}^{t}\left(\operatorname{div}_{h}\left(\bar{u}_{N} \otimes \bar{u}_{N}\right)\left(t^{\prime}\right) \mid u_{\mathrm{app}, N}^{\varepsilon}\left(t^{\prime}\right)\right)_{L^{2}} \mathrm{~d} t^{\prime}=\int_{0}^{t}\left(\operatorname{div}_{h}\left(\bar{u}_{N} \otimes \bar{u}_{N}\right)\left(t^{\prime}\right) \mid \bar{u}_{N}\left(t^{\prime}\right)\right)_{L^{2}} \mathrm{~d} t^{\prime} & \\
& +\int_{0}^{t}\left(\operatorname{div}_{h}\left(\bar{u}_{N} \otimes \bar{u}_{N}\right)\left(t^{\prime}\right) \mid \mathcal{L}\left(\frac{t^{\prime}}{\varepsilon}\right) v_{N}\left(t^{\prime}\right)\right)_{L^{2}} \mathrm{~d} t^{\prime}+\rho_{N}^{\varepsilon} .
\end{aligned}
$$

But $\bar{u}_{N}$ (and thus $\operatorname{div}_{h}\left(\bar{u}_{N} \otimes \bar{u}_{N}\right)$ ) is a horizontal vector field, and $\operatorname{since} \operatorname{div}_{h} \bar{u}_{N}=0$, we have

$$
\int_{0}^{t}\left(\operatorname{div}_{h}\left(\bar{u}_{N} \otimes \bar{u}_{N}\right)\left(t^{\prime}\right) \mid \bar{u}_{N}\left(t^{\prime}\right)\right)_{L^{2}} \mathrm{~d} t^{\prime}=0 .
$$

Moreover, by definition of $v_{N}$ and $\mathcal{L}(\tau)$, we have

$$
\begin{aligned}
\left(\operatorname{div}_{h}\left(\bar{u}_{N} \otimes \bar{u}_{N}\right)(t) \mid \mathcal{L}\left(\frac{t}{\varepsilon}\right)\right. & \left.v_{N}\left(t^{\prime}\right)\right)_{L^{2}} \\
& \sum_{k=1}^{N} \int_{\Omega} \operatorname{div}_{h}\left(\bar{u}_{N} \otimes \bar{u}_{N}\right)(t) \cdot \mathcal{F}^{-1} A\left(\xi_{h}\right) \mathcal{L}_{k}\left(\frac{t}{\varepsilon}\right) A^{-1}\left(\xi_{h}\right) \widehat{v}^{k, h}\left(\xi_{h}\right)\left(t^{\prime}\right) \cos \left(k \pi x_{3}\right) \mathrm{d} x_{h} \mathrm{~d} x_{3} .
\end{aligned}
$$


But as $\int_{0}^{1} \cos \left(k \pi x_{3}\right) \mathrm{d} x_{3}=0$, if $k \neq 0$, we have

$$
\int_{0}^{t}\left(\operatorname{div}_{h}\left(\bar{u}_{N} \otimes \bar{u}_{N}\right)\left(t^{\prime}\right) \mid \mathcal{L}\left(\frac{t^{\prime}}{\varepsilon}\right) v_{N}\left(t^{\prime}\right)\right)_{L^{2}} \mathrm{~d} t^{\prime}=0
$$

so finally

$$
\int_{0}^{t}\left(\operatorname{div}_{h}\left(\bar{u}_{N} \otimes \bar{u}_{N}\right)\left(t^{\prime}\right) \mid u_{\mathrm{app}, N}^{\varepsilon}\left(t^{\prime}\right)\right)_{L^{2}} \mathrm{~d} t^{\prime}=\rho_{N}^{\varepsilon}
$$

As an immediate consequence, it turns out that

$$
\left\|u_{\mathrm{app}, N}^{\varepsilon}(t)\right\|_{L^{2}}^{2}+2 \nu \int_{0}^{t}\left\|\nabla^{h} u_{\mathrm{app}, N}^{\varepsilon}\left(t^{\prime}\right)\right\|_{L^{2}}^{2} \mathrm{~d} t^{\prime}+2 \beta \varepsilon \int_{0}^{t}\left\|\partial_{3} u_{\mathrm{app}, N}^{\varepsilon}\left(t^{\prime}\right)\right\|_{L^{2}}^{2} \mathrm{~d} t^{\prime} \leq\left\|u_{0}\right\|_{L^{2}}^{2}+\rho_{N}^{\varepsilon} .
$$

Equation (3.5) can be rewritten as

$$
L^{\varepsilon} u_{\mathrm{app}, N}^{\varepsilon}+u_{\mathrm{app}, N}^{\varepsilon} \cdot \nabla u_{\mathrm{app}, N}^{\varepsilon}=R_{N}^{\varepsilon}+\nabla p_{\varepsilon}+F_{N}^{\varepsilon}
$$

with

$$
F_{N}^{\varepsilon} \stackrel{\text { def }}{=} u_{\mathrm{app}, N}^{\varepsilon} \cdot \nabla u_{\mathrm{app}, N}^{\varepsilon}-\bar{u}_{N} \cdot \nabla \bar{u}_{N}
$$

Now, we use the classical method to prove weak strong type estimates. We are exactly in this situation because we consider a weak solution $u^{\varepsilon}$ without any additional regularity and a regular (approximate) solution $u_{\text {app }, N}^{\varepsilon}$. Let us denote by $\delta^{\varepsilon}$ the difference $u^{\varepsilon}-u_{\text {app }, N}^{\varepsilon}$, we write that

$$
\begin{aligned}
E^{\varepsilon}(t) \stackrel{\text { def }}{=} & \left\|\delta^{\varepsilon}(t)\right\|_{L^{2}}^{2}+2 \nu \int_{0}^{t}\left\|\nabla^{h} \delta^{\varepsilon}\left(t^{\prime}\right)\right\|_{L^{2}}^{2} \mathrm{~d} t^{\prime}+2 \beta \varepsilon \int_{0}^{t}\left\|\partial_{3} \delta^{\varepsilon}\left(t^{\prime}\right)\right\|_{L^{2}}^{2} \mathrm{~d} t^{\prime} \\
= & \left\|u^{\varepsilon}(t)\right\|_{L^{2}}^{2}+2 \nu \int_{0}^{t}\left\|\nabla^{h} u^{\varepsilon}\left(t^{\prime}\right)\right\|_{L^{2}}^{2} \mathrm{~d} t^{\prime}+2 \beta \varepsilon \int_{0}^{t}\left\|\partial_{3} u^{\varepsilon}\left(t^{\prime}\right)\right\|_{L^{2}}^{2} \mathrm{~d} t^{\prime} \\
& +\left\|u_{\mathrm{app}, N}^{\varepsilon}(t)\right\|_{L^{2}}^{2}+2 \nu \int_{0}^{t}\left\|\nabla^{h} u_{\mathrm{app}, N}^{\varepsilon}\left(t^{\prime}\right)\right\|_{L^{2}}^{2} \mathrm{~d} t^{\prime}+2 \beta \varepsilon \int_{0}^{t}\left\|\partial_{3} u_{\mathrm{app}, N}^{\varepsilon}\left(t^{\prime}\right)\right\|_{L^{2}}^{2} \mathrm{~d} t^{\prime} \\
& -2\left(u^{\varepsilon}(t) \mid u_{\mathrm{app}, N}^{\varepsilon}(t)\right)_{L^{2}}-4 \nu \int_{0}^{t}\left(\nabla^{h} u^{\varepsilon}\left(t^{\prime}\right) \mid \nabla^{h} u_{\mathrm{app}, N}^{\varepsilon}\left(t^{\prime}\right)\right)_{L^{2}} \mathrm{~d} t^{\prime} \\
& -4 \beta \varepsilon \int_{0}^{t}\left(\partial_{3} u^{\varepsilon}\left(t^{\prime}\right) \mid \partial_{3} u_{\mathrm{app}, N}^{\varepsilon}\left(t^{\prime}\right)\right)_{L^{2}} \mathrm{~d} t^{\prime} .
\end{aligned}
$$

As $u^{\varepsilon}$ is a Leray solution of $\left(R F_{\varepsilon}\right)$, it satisfies the energy inequality. So thanks to inequality (3.6) we get

$$
\begin{array}{r}
E^{\varepsilon}(t) \leq 2\left\|u_{0}\right\|_{L^{2}}^{2}-2\left(u^{\varepsilon} \mid u_{\mathrm{app}, N}^{\varepsilon}\right)_{L^{2}}-4 \nu \int_{0}^{t}\left(\nabla^{h} u^{\varepsilon}\left(t^{\prime}\right) \mid \nabla^{h} u_{\mathrm{app}, N}^{\varepsilon}\left(t^{\prime}\right)\right)_{L^{2}} \mathrm{~d} t^{\prime} \\
-4 \beta \varepsilon \int_{0}^{t}\left(\partial_{3} u^{\varepsilon}\left(t^{\prime}\right) \mid \partial_{3} u_{\mathrm{app}, N}^{\varepsilon}\left(t^{\prime}\right)\right)_{L^{2}} \mathrm{~d} t^{\prime}+\rho_{N}^{\varepsilon} .
\end{array}
$$

As $u_{\mathrm{app}, N}^{\varepsilon}$ is a smooth function such that $u_{\mathrm{app}, N}^{\varepsilon}{ }_{\mid \partial \Omega}=\partial_{t} u_{\mathrm{app}, N \mid \partial \Omega}^{\varepsilon}=0$, the function

$$
t \mapsto\left(u^{\varepsilon}(t) \mid u_{\mathrm{app}, N}^{\varepsilon}(t)\right)_{L^{2}}
$$


is $C^{1}$, so we can write, using (3.7) and the fact that $a \times e_{3} \cdot b+b \times e_{3} \cdot a=0$,

$$
\begin{aligned}
\frac{\mathrm{d}}{\mathrm{d} t}\left(u^{\varepsilon} \mid u_{\mathrm{app}, N}^{\varepsilon}\right)_{L^{2}}=\left(\nu \Delta_{h} u^{\varepsilon}+\beta \varepsilon \partial_{3}^{2} u^{\varepsilon}-\right. & \left.u^{\varepsilon} \cdot \nabla u^{\varepsilon}-\nabla p^{\varepsilon} \mid u_{\mathrm{app}, N}^{\varepsilon}\right)_{L^{2}} \\
& +\left(\nu \Delta_{h} u_{\mathrm{app}, N}^{\varepsilon}+\beta \varepsilon \partial_{3}^{2} u_{\mathrm{app}, N}^{\varepsilon}-u_{\mathrm{app}, N}^{\varepsilon} \cdot \nabla u_{\mathrm{app}, N}^{\varepsilon}-\nabla p_{\mathrm{app}}^{\varepsilon}+F_{N}^{\varepsilon} \mid u^{\varepsilon}\right)_{L^{2}} .
\end{aligned}
$$

By an integration by parts allowed by the fact that $u^{\varepsilon}$ and $u_{\mathrm{app}, N}^{\varepsilon}$ vanish at the boundary, we have

$$
\begin{aligned}
\frac{\mathrm{d}}{\mathrm{d} t}\left(u^{\varepsilon} \mid u_{\mathrm{app}, N}^{\varepsilon}\right)_{L^{2}}=-2 \nu\left(\nabla^{h} u^{\varepsilon} \mid \nabla^{h} u_{\mathrm{app}, N}^{\varepsilon}\right)_{L^{2}}-2 \beta \varepsilon\left(\partial_{3} u^{\varepsilon} \mid \partial_{3} u_{\mathrm{app}, N}^{\varepsilon}\right)_{L^{2}} \\
-\left(u^{\varepsilon} \cdot \nabla u^{\varepsilon} \mid u_{\mathrm{app}, N}^{\varepsilon}\right)_{L^{2}}-\left(u_{\mathrm{app}, N}^{\varepsilon} \cdot \nabla u_{\mathrm{app}, N}^{\varepsilon} \mid u^{\varepsilon}\right)_{L^{2}}+\left(F_{N}^{\varepsilon} \mid u^{\varepsilon}\right)_{L^{2}} .
\end{aligned}
$$

The fact that $u_{\mathrm{app}, N}^{\varepsilon}$ is a smooth function and that $u^{\varepsilon}$ and $u_{\mathrm{app}, N}^{\varepsilon}$ are divergence free vector fields that vanish on the boundary imply that

$$
\left(u^{\varepsilon} \cdot \nabla u^{\varepsilon} \mid u_{\mathrm{app}, N}^{\varepsilon}\right)_{L^{2}}+\left(u_{\mathrm{app}, N}^{\varepsilon} \cdot \nabla u_{\mathrm{app}, N}^{\varepsilon} \mid u^{\varepsilon}\right)_{L^{2}}=\left(\delta^{\varepsilon} \cdot \nabla \delta^{\varepsilon} \mid u_{\mathrm{app}, N}^{\varepsilon}\right)_{L^{2}} .
$$

Using again the fact that $u_{\mathrm{app}, N}^{\varepsilon}$ is a smooth function and that $u^{\varepsilon}$ and $u_{\mathrm{app}, N}^{\varepsilon}$ are divergence free vector fields that vanish on the boundary, we infer that

$$
\frac{\mathrm{d}}{\mathrm{d} t}\left(u^{\varepsilon} \mid u_{\mathrm{app}, N}^{\varepsilon}\right)_{L^{2}}=-2 \nu\left(\nabla^{h} u^{\varepsilon} \mid \nabla^{h} u_{\mathrm{app}, N}^{\varepsilon}\right)_{L^{2}}-2 \beta \varepsilon\left(\partial_{3} u^{\varepsilon} \mid \partial_{3} u_{\mathrm{app}, N}^{\varepsilon}\right)_{L^{2}}-\left(\delta^{\varepsilon} \cdot \nabla \delta^{\varepsilon} \mid u_{\mathrm{app}, N}^{\varepsilon}\right)_{L^{2}}+\left(F_{N}^{\varepsilon} \mid u^{\varepsilon}\right)_{L^{2}} .
$$

By integration and using Lemmas 1 and 2 we get that

$$
\begin{aligned}
\left(u^{\varepsilon} \mid u_{\mathrm{app}, N}^{\varepsilon}\right)_{L^{2}}(t)= & \left\|u_{0}\right\|_{L^{2}}^{2}+\rho_{N}^{\varepsilon} \\
& -2 \nu \int_{0}^{t}\left(\nabla^{h} u^{\varepsilon}\left(t^{\prime}\right) \mid \nabla^{h} u_{\mathrm{app}, N}^{\varepsilon}\left(t^{\prime}\right)\right)_{L^{2}} \mathrm{~d} t^{\prime}-2 \beta \varepsilon \int_{0}^{t}\left(\partial_{3} u^{\varepsilon}\left(t^{\prime}\right) \mid \partial_{3} u_{\mathrm{app}, N}^{\varepsilon}\left(t^{\prime}\right)\right)_{L^{2}} \mathrm{~d} t^{\prime} \\
& -\int_{0}^{t}\left(\delta^{\varepsilon}\left(t^{\prime}\right) \cdot \nabla \delta^{\varepsilon}\left(t^{\prime}\right) \mid u_{\mathrm{app}, N}^{\varepsilon}\left(t^{\prime}\right)\right)_{L^{2}} \mathrm{~d} t^{\prime}+\int_{0}^{t}\left(F_{N}^{\varepsilon}\left(t^{\prime}\right) \mid u^{\varepsilon}\left(t^{\prime}\right)\right)_{L^{2}} \mathrm{~d} t^{\prime} .
\end{aligned}
$$

So we infer that

$$
E^{\varepsilon}(t) \leq 2 \int_{0}^{t}\left(\delta^{\varepsilon}\left(t^{\prime}\right) \cdot \nabla \delta^{\varepsilon}\left(t^{\prime}\right) \mid u_{\mathrm{app}, N}^{\varepsilon}\left(t^{\prime}\right)\right)_{L^{2}} \mathrm{~d} t^{\prime}+2 \int_{0}^{t}\left(F_{N}^{\varepsilon}\left(t^{\prime}\right) \mid u^{\varepsilon}\left(t^{\prime}\right)\right)_{L^{2}} \mathrm{~d} t^{\prime}+\rho_{N}^{\varepsilon} .
$$

Now let us state the lemmas which will enable us to conclude the proof of Theorem 2 .

Lemma 4. Let $u$ (resp. $v$ ) be any vector field (resp. divergence free vector field) in $H^{2}(\Omega)$ (resp. $H_{0}^{1}(\Omega)$ ). If we define

$$
N(u)^{2} \stackrel{\text { def }}{=} \sup _{x_{3} \in[0,1]}\left\|\nabla^{h} u\left(\cdot, x_{3}\right)\right\|_{L^{2}\left(\mathbf{R}^{2}\right)}^{2}+\int_{0}^{1} \mathrm{~d}\left(x_{3}\right)\left\|\partial_{3} u\left(\cdot, x_{3}\right)\right\|_{L^{4}\left(\mathbf{R}^{2}\right)}^{2} \mathrm{~d} x_{3},
$$

then we have

$$
(v \cdot \nabla v \mid u)_{L^{2}} \leq \frac{\nu}{4}\left\|\nabla^{h} v\right\|_{L^{2}}^{2}+\frac{C}{\nu} N(u)^{2}\|v\|_{L^{2}}^{2} .
$$

In the following lemma, and subsequently, we note $O_{N}\left(\varepsilon^{\frac{1}{2}}\right)$ a quantity of the order of magnitude $\varepsilon^{\frac{1}{2}}$, depending on $N$. 
Lemma 5. We have

$$
\int_{0}^{t}\left(F_{N}^{\varepsilon}\left(t^{\prime}\right) \mid u^{\varepsilon}\left(t^{\prime}\right)\right)_{L^{2}} \mathrm{~d} t^{\prime}=O_{N}\left(\varepsilon^{\frac{1}{2}}\right)
$$

The proof of this lemma uses in a crucial way dispersive estimates which are summarized in the following lemma:

Lemma 6. Let $p \in[1,+\infty]$ be given, and let us denote by

$$
u_{0, \text { int }, N} \stackrel{\text { def }}{=} \mathcal{L}\left(\frac{t}{\varepsilon}\right) u_{N}
$$

where $u_{N}$ is the solution of

$$
\partial_{t} u_{N}-\nu \Delta_{h} u_{N}+\mathcal{E} u_{N}=0
$$

with the initial data $u_{0, N}$. Then we have

$$
\left\|u_{0, \text { int }, N}^{\varepsilon}-\bar{u}_{N}\right\|_{L^{p}\left(\mathbf{R}^{+} ; L^{\infty}(\Omega)\right)} \leq C_{N} \varepsilon^{\frac{1}{2}-\frac{1}{4 p}}\left\|u_{0}\right\|_{L^{2}} .
$$

The following corollary is the result of an easy interpolation.

Corollary 1. For any $p \in[1,+\infty]$, any $\alpha>0$ and any $q \in] 2,+\infty]$, we have

$$
\left\|\partial^{\alpha}\left(u_{0, \text { int }, N}^{\varepsilon}-\bar{u}_{N}\right)\right\|_{L^{p}\left(\mathbf{R}^{+} ; L^{q}(\Omega)\right)}=\rho_{N}^{\varepsilon} .
$$

Let us postpone the proof of those lemmas to the next paragraph and to Section 4 . They imply that

$$
E^{\varepsilon}(t) \leq \frac{\nu}{2} \int_{0}^{t}\left\|\nabla^{h} \delta^{\varepsilon}\left(t^{\prime}\right)\right\|_{L^{2}}^{2} \mathrm{~d} t^{\prime}+\frac{C}{\nu} \int_{0}^{t} N\left(u_{\mathrm{app}, N}^{\varepsilon}\left(t^{\prime}\right)\right)^{2}\left\|\delta^{\varepsilon}\left(t^{\prime}\right)\right\|_{L^{2}}^{2} \mathrm{~d} t^{\prime}+\rho_{N}^{\varepsilon} .
$$

But Lemmas 1 and 2 imply in particular that there is a constant $C_{N}$ such that for any $\varepsilon$,

$$
\int_{0}^{\infty} N\left(u_{\mathrm{app}, N}^{\varepsilon}(t)\right)^{2} \mathrm{~d} t \leq C_{N}\left\|u_{0}\right\|_{L^{2}}^{2}
$$

So a Gronwall lemma implies that

$$
\sup _{t \geq 0}\left\|u^{\varepsilon}(t)-u_{\mathrm{app}, N}^{\varepsilon}(t)\right\|_{L^{2}}^{2}+2 \nu \int_{0}^{\infty}\left\|\nabla^{h}\left(u^{\varepsilon}\left(t^{\prime}\right)-u_{\mathrm{app}, N}^{\varepsilon}\left(t^{\prime}\right)\right)\right\|_{L^{2}}^{2} \mathrm{~d} t^{\prime}=\rho_{N}^{\varepsilon} .
$$

Now we can conclude the proof of Theorem 2. From (2.7) we infer that

$$
\sup _{t \geq 0}\left\|u^{\varepsilon}(t)-u_{0, \text { int }, N}^{\varepsilon}(t)\right\|_{L^{2}}^{2}+2 \nu \int_{0}^{\infty}\left\|\nabla^{h}\left(u^{\varepsilon}-u_{0, \text { int }, N}^{\varepsilon}\right)\left(t^{\prime}\right)\right\| \mathrm{d} t^{\prime}=\rho_{N}^{\varepsilon} .
$$

Thanks to the above corollary, we infer that for any compact subset $K$ of $\Omega$, we have

$$
\sup _{t \geq 0}\left\|u^{\varepsilon}(t)-\bar{u}_{N}(t)\right\|_{L^{2}}^{2}+2 \nu \int_{0}^{\infty}\left\|\nabla^{h}\left(u^{\varepsilon}-\bar{u}_{N}\right)\left(t^{\prime}\right)\right\| \mathrm{d} t^{\prime}=\rho_{N}^{\varepsilon} .
$$

The result follows from the fact that $\bar{u}_{N}$ converges towards $\bar{u}$ in the energy space, as $N$ goes to infinity. 
Now let us prove the lemmas we have admitted above, starting with Lemma 4 . We have to estimate

$$
I_{j, k} \stackrel{\text { def }}{=} \int_{\Omega} v^{j}(x) v^{k}(x) \partial_{k} u^{j}(x) \mathrm{d} x .
$$

The case when $k \neq 3$ is easier. Let us write that

$$
\left|I_{j, k}\right| \leq \int_{0}^{1}\left\|v\left(\cdot, x_{3}\right)\right\|_{L^{4}\left(\mathbf{R}^{2}\right)}^{2}\left\|\nabla^{h} u\left(\cdot, x_{3}\right)\right\|_{L^{2}\left(\mathbf{R}^{2}\right)} \mathrm{d} x_{3} .
$$

Using the fact that $\|a\|_{L^{4}\left(\mathbf{R}^{2}\right)}^{2} \leq\|a\|_{L^{2}\left(\mathbf{R}^{2}\right)}\left\|\nabla^{h} a\right\|_{L^{2}\left(\mathbf{R}^{2}\right)}$, we have by definition of $N(u)$,

$$
\begin{aligned}
\left|I_{j, k}\right| & \leq \int_{0}^{1}\left\|v\left(\cdot, x_{3}\right)\right\|_{L^{2}\left(\mathbf{R}^{2}\right)}\left\|\nabla^{h} v\left(\cdot, x_{3}\right)\right\|_{L^{2}\left(\mathbf{R}^{2}\right)}\left\|\nabla^{h} u\left(\cdot, x_{3}\right)\right\|_{L^{2}\left(\mathbf{R}^{2}\right)} \mathrm{d} x_{3} \\
& \leq N(u) \int_{0}^{1}\left\|v\left(\cdot, x_{3}\right)\right\|_{L^{2}\left(\mathbf{R}^{2}\right)}\left\|\nabla^{h} v\left(\cdot, x_{3}\right)\right\|_{L^{2}\left(\mathbf{R}^{2}\right)} \mathrm{d} x_{3} \\
& \leq N(u)\|v\|_{L^{2}}\left\|\nabla^{h} v\right\|_{L^{2}} .
\end{aligned}
$$

So Lemma 4 is proved for $I_{j, k}$ which $k \neq 3$. When $k=3$, we shall use the following lemma:

Lemma 7. Let $v$ be a divergence free vector field in $H_{0}^{1}(\Omega)$. Then we have for almost every $x_{h} \in \mathbf{R}^{2}$

$$
\sup _{\left.x_{3} \in\right] 0,1[} \frac{\left|v^{3}\left(x_{h}, x_{3}\right)\right|}{d\left(x_{3}\right)^{\frac{1}{2}}} \leq\left\|\operatorname{div}_{h} v^{h}\left(x_{h}, \cdot\right)\right\|_{L^{2}(] 0,1[)} .
$$

Let us prove Lemma 7. As $v$ is a smooth divergence free vector field whose value on the boundary is 0 , we have

$$
\begin{aligned}
v^{3}\left(x_{h}, x_{3}\right) & =\int_{0}^{x_{3}} \partial_{3} v^{3}\left(x_{h}, y_{3}\right) \mathrm{d} y_{3} \\
& =-\int_{0}^{x_{3}} \operatorname{div}_{h} v^{h}\left(x_{h}, y_{3}\right) \mathrm{d} y_{3} .
\end{aligned}
$$

Using Cauchy-Schwarz inequality, we have

$$
\left|v^{3}\left(x_{h}, x_{3}\right)\right|^{2} \leq x_{3} \int_{0}^{1}\left|\operatorname{div}_{h} v^{h}\left(x_{h}, y_{3}\right)\right|^{2} \mathrm{~d} y_{3} .
$$

As the same is true for the upper boundary, Lemma 7 is proved.

Lemma 7 implies that

$$
\left.\forall x_{3} \in\right] 0,1\left[,\left\|v\left(\cdot, x_{3}\right)\right\|_{L^{2}\left(\mathbf{R}^{2}\right)} \leq \mathrm{d}\left(x_{3}\right)^{\frac{1}{2}}\left\|\operatorname{div}_{h} v^{h}\right\|_{L^{2}} .\right.
$$

Now let us write that

$$
\begin{aligned}
\left|I_{j, 3}\right| & \leq \int_{0}^{1}\left\|v\left(\cdot, x_{3}\right)\right\|_{L^{4}\left(\mathbf{R}^{2}\right)}\left\|v^{3}\left(\cdot, x_{3}\right)\right\|_{L^{2}\left(\mathbf{R}^{2}\right)}\left\|\partial_{3} u\left(\cdot, x_{3}\right)\right\|_{L^{4}\left(\mathbf{R}^{2}\right)} \mathrm{d} x_{3} \\
& \leq\left\|\operatorname{div}_{h} v^{h}\right\|_{L^{2}} \int_{0}^{1}\left\|v\left(\cdot, x_{3}\right)\right\|_{L^{4}\left(\mathbf{R}^{2}\right)} d\left(x_{3}\right)^{\frac{1}{2}}\left\|\partial_{3} u\left(\cdot, x_{3}\right)\right\|_{L^{4}\left(\mathbf{R}^{2}\right)} \mathrm{d} x_{3} .
\end{aligned}
$$


Using Cauchy-Schwarz and Sobolev inequalities, that inequality implies that

$$
\begin{aligned}
\left|I_{j, 3}\right| & \leq\left\|\operatorname{div}_{h} v^{h}\right\|_{L^{2}}\left(\int_{0}^{1}\left\|v\left(\cdot, x_{3}\right)\right\|_{L^{4}\left(\mathbf{R}^{2}\right)}^{2} \mathrm{~d} x_{3}\right)^{\frac{1}{2}}\left(\int_{0}^{1} \mathrm{~d}\left(x_{3}\right)\left\|\partial_{3} u\left(\cdot, x_{3}\right)\right\|_{L^{4}\left(\mathbf{R}^{2}\right)}^{2} \mathrm{~d} x_{3}\right)^{\frac{1}{2}} \\
& \leq\left\|\nabla^{h} v\right\|_{L^{2}}^{\frac{3}{2}}\|v\|_{L^{2}}^{\frac{1}{2}} N(u) .
\end{aligned}
$$

Using a log-concavity inequality, Lemma 4 is proved.

Now let us prove Lemma 5. To begin with let us decompose $F_{N}^{\varepsilon}$ as

$$
\begin{aligned}
& F_{N}^{\varepsilon}=F_{N, 1}^{\varepsilon}+F_{N, 2}^{\varepsilon} \quad \text { with } \\
& F_{N, 1}^{\varepsilon} \stackrel{\text { def }}{=} u_{\mathrm{app}, N}^{\varepsilon} \cdot \nabla u_{\mathrm{app}, N}^{\varepsilon}-u_{0, \text { int }, N}^{\varepsilon} \cdot \nabla u_{0, \text { int }, N}^{\varepsilon} \quad \text { and } \\
& F_{N, 2}^{\varepsilon} \stackrel{\text { def }}{=} u_{0, \text { int }, N}^{\varepsilon} \cdot \nabla u_{0, \text { int }, N}^{\varepsilon}-\bar{u}_{N} \cdot \nabla \bar{u}_{N} .
\end{aligned}
$$

These two terms will be estimated in two different ways. The estimate on $F_{N, 1}^{\varepsilon}$ does not use any dispersion estimates but the one on $F_{N, 2}^{\varepsilon}$ does indeed. To estimate $F_{N, 1}^{\varepsilon}$, let us write

$$
\begin{aligned}
& F_{N, 1}^{\varepsilon}=F_{N, 11}^{\varepsilon, h}+F_{N, 12}^{\varepsilon, h}+F_{N, 11}^{\varepsilon, 3}+F_{N, 12}^{\varepsilon, 3} \text { with } \\
& F_{N, 11}^{\varepsilon, h} \stackrel{\text { def }}{=}\left(u_{\text {app }, N}^{\varepsilon, h}-u_{0, \text { int }, N}^{\varepsilon, h}\right) \cdot \nabla^{h} u_{\text {app }, N}^{\varepsilon} \\
& F_{N, 12}^{\varepsilon, h} \stackrel{\text { def }}{=} u_{0, \text { int }, N}^{\varepsilon, h} \cdot \nabla^{h}\left(u_{\text {app }, N}^{\varepsilon}-u_{0, \text { int }, N}^{\varepsilon}\right) \\
& F_{N, 11}^{\varepsilon, 3} \stackrel{\text { def }}{=}\left(u_{\text {app }, N}^{\varepsilon, 3}-u_{0, \text { int }, N}^{\varepsilon, 3}\right) \partial_{3} u_{\text {app }, N}^{\varepsilon} \quad \text { and } \\
& F_{N, 12}^{\varepsilon, 3} \stackrel{\text { def }}{=} u_{0, \text { int }, N}^{\varepsilon, 3} \partial_{3}\left(u_{\text {app }, N}^{\varepsilon}-u_{0, \text { int }, N}^{\varepsilon}\right) .
\end{aligned}
$$

As usual, terms which do not contain vertical derivatives are easier to deal with. Using Cauchy-Schwarz and Sobolev inequalities, we get

$$
\begin{aligned}
\left|\left(F_{N, 11}^{\varepsilon, h} \mid u^{\varepsilon}\right)_{L^{2}}\right| \leq & \sup _{\left.x_{3} \in\right] 0,1[}\left\|\nabla^{h} u_{\mathrm{app}, N}^{\varepsilon}\right\|_{L^{2}\left(\mathbf{R}^{2}\right)} \\
& \times \int_{0}^{1}\left\|\left(u_{\mathrm{app}, N}^{\varepsilon}-u_{0, \mathrm{int}, N}^{\varepsilon}\right)\left(\cdot, x_{3}\right)\right\|_{L^{4}\left(\mathbf{R}^{2}\right)}\left\|u^{\varepsilon}\left(\cdot, x_{3}\right)\right\|_{L^{4}\left(\mathbf{R}^{2}\right)} \mathrm{d} x_{3} \\
\leq & N\left(u_{\mathrm{app}, N}^{\varepsilon}\right) \int_{0}^{1}\left\|\nabla^{h}\left(u_{\mathrm{app}, N}^{\varepsilon}-u_{0, \mathrm{int}, N}^{\varepsilon}\right)\left(\cdot, x_{3}\right)\right\|_{L^{2}\left(\mathbf{R}^{2}\right)}^{\frac{1}{2}} \\
& \times\left\|u_{\mathrm{app}, N}^{\varepsilon}-u_{0, \mathrm{int}, N}^{\varepsilon}\left(\cdot, x_{3}\right)\right\|_{L^{2}\left(\mathbf{R}^{2}\right)}^{\frac{1}{2}}\left\|\nabla^{h} u^{\varepsilon}\left(\cdot, x_{3}\right)\right\|_{L^{2}\left(\mathbf{R}^{2}\right)}^{\frac{1}{2}}\left\|u^{\varepsilon}\left(\cdot, x_{3}\right)\right\|_{L^{2}\left(\mathbf{R}^{2}\right)}^{\frac{1}{2}} \mathrm{~d} x_{3} .
\end{aligned}
$$

Using estimates (2.7) and (2.8) of Lemma 2, we have that

$$
\int_{0}^{\infty}\left|\left(F_{N, 11}^{\varepsilon, h}(t) \mid u^{\varepsilon}(t)\right)_{L^{2}}\right| \mathrm{d} t \leq C_{N^{\varepsilon^{2}}}{ }^{\frac{1}{2}}\left\|u_{0}\right\|_{L^{2}}^{2}
$$


Note that $E(T)$ is bounded uniformly in time. The estimate about $F_{N, 12}^{\varepsilon, h}$ will be proved following the same lines. We have

$$
\begin{aligned}
\left|\left(F_{N, 12}^{\varepsilon, h} \mid u^{\varepsilon}\right)_{L^{2}}\right| \leq & \sup _{\left.x_{3} \in\right] 0,1[}\left\|u_{0, \text { int }, N}^{\varepsilon}\left(\cdot, x_{3}\right)\right\|_{L^{4}\left(\mathbf{R}^{2}\right)} \\
& \times \int_{0}^{1}\left\|\nabla^{h}\left(u_{\mathrm{app}, N}^{\varepsilon}-u_{0, \text { int }, N}^{\varepsilon}\right)\left(\cdot, x_{3}\right)\right\|_{L^{2}\left(\mathbf{R}^{2}\right)}\left\|u^{\varepsilon}\right\|_{L^{4}\left(\mathbf{R}^{2}\right)} \mathrm{d} x_{3} \\
\leq & C_{N}\left\|u_{0}\right\|_{L^{2}} N\left(u_{0, \text { int }, N}^{\varepsilon}\right)^{\frac{1}{2}}\left\|\nabla^{h}\left(u_{\mathrm{app}, N}^{\varepsilon}-u_{0, \text { int }, N}^{\varepsilon}\right)\right\|_{L^{2}}\left\|\nabla^{h} u^{\varepsilon}\right\|_{L^{2}}^{\frac{1}{2}} .
\end{aligned}
$$

So it turns out that

$$
\int_{0}^{\infty}\left|\left(F_{N, 12}^{\varepsilon, h}(t) \mid u^{\varepsilon}(t)\right)_{L^{2}}\right| \mathrm{d} t \leq C_{N} \varepsilon^{\frac{1}{2}}\left\|u_{0}\right\|_{L^{2}}^{2} .
$$

As it is now usual, terms which contain vertical derivatives are estimated in another way. Using Lemma 7, we have

$$
\left\|\left(u_{\mathrm{app}, N}^{\varepsilon, 3}-u_{0, \text { int }, N}^{\varepsilon, 3}\right)\left(\cdot, x_{3}\right)\right\|_{L^{2}\left(\mathbf{R}^{2}\right)} \leq \mathrm{d}\left(x_{3}\right)^{\frac{1}{2}}\left\|\nabla^{h}\left(u_{\mathrm{app}, N}^{\varepsilon}-u_{0, \text { int }, N}^{\varepsilon}\right)\right\|_{L^{2}} .
$$

So we have

$$
\left|\left(F_{N, 11}^{\varepsilon, 3} \mid u^{\varepsilon}\right)_{L^{2}}\right| \leq\left\|\nabla^{h}\left(u_{\mathrm{app}, N}^{\varepsilon}-u_{0, \mathrm{int}, N}^{\varepsilon}\right)\right\|_{L^{2}} \int_{0}^{1} \mathrm{~d}\left(x_{3}\right)^{\frac{1}{2}}\left\|\partial_{3} u_{\mathrm{app}, N}^{\varepsilon}\left(\cdot, x_{3}\right)\right\|_{L^{\infty}\left(\mathbf{R}^{2}\right)}\left\|u^{\varepsilon}\left(\cdot, x_{3}\right)\right\|_{L^{2}\left(\mathbf{R}^{2}\right)} \mathrm{d} x_{3} .
$$

By Cauchy-Schwarz inequality, we have that

$$
\left|\left(F_{N, 11}^{\varepsilon, 3} \mid u^{\varepsilon}\right)_{L^{2}}\right| \leq\left\|\nabla^{h}\left(u_{\mathrm{app}, N}^{\varepsilon}-u_{0, \text { int }, N}^{\varepsilon}\right)\right\|_{L^{2}}\left(\int_{0}^{1} \mathrm{~d}\left(x_{3}\right)\left\|\partial u_{\mathrm{app}, N}^{\varepsilon}\left(\cdot, x_{3}\right)\right\|_{L^{\infty}\left(\mathbf{R}^{2}\right)} \mathrm{d} x_{3}\right)^{\frac{1}{2}}\left\|u^{\varepsilon}\right\|_{L^{2}} .
$$

So applying inequalities (2.7) and (2.9) of Lemma 2 we get that

$$
\int_{0}^{\infty}\left|\left(F_{N, 11}^{\varepsilon, 3}(t) \mid u^{\varepsilon}(t)\right)_{L^{2}}\right| \mathrm{d} t \leq C_{N} \varepsilon^{\frac{1}{2}}\left\|u_{0}\right\|_{L^{2}}^{2} .
$$

To estimate the term $F_{N, 12}^{\varepsilon, 3}$, let us observe that

$$
u_{0, \text { int }, N}^{\varepsilon, 3}\left(x_{h}, x_{3}\right)=-\sum_{k=1}^{N} \frac{1}{k \pi} \operatorname{div}_{h} u_{0, \text { int }, N}^{\varepsilon, k, h}\left(x_{h}\right) \sin \left(k \pi x_{3}\right) .
$$

So it turns out that

$$
\left\|u_{0, \text { int }, N}^{\varepsilon, 3}\left(\cdot, x_{3}\right)\right\|_{L^{2}\left(\mathbf{R}^{2}\right)} \leq C_{N} \mathrm{~d}\left(x_{3}\right)\left\|\nabla^{h} u_{0, \text { int }, N}^{\varepsilon}\right\|_{L^{2}}
$$

So we find

$$
\left|\left(F_{N, 12}^{\varepsilon, 3} \mid u^{\varepsilon}\right)_{L^{2}}\right| \leq\left\|\nabla^{h} u_{0, \text { int }, N}^{\varepsilon}\right\|_{L^{2}} \int_{0}^{1} \mathrm{~d}\left(x_{3}\right)\left\|\partial_{3}\left(u_{\mathrm{app}, N}^{\varepsilon}-u_{0, \text { int }, N}^{\varepsilon}\right)\left(\cdot, x_{3}\right)\right\|_{L^{\infty}\left(\mathbf{R}^{2}\right)}\left\|u^{\varepsilon}\left(\cdot, x_{3}\right)\right\|_{L^{2}\left(\mathbf{R}^{2}\right)} \mathrm{d} x_{3} .
$$

So by Cauchy-Schwarz inequality, we get

$$
\left|\left(F_{N, 12}^{\varepsilon, 3} \mid u^{\varepsilon}\right)_{L^{2}}\right| \leq\left\|\nabla^{h} u_{0, \text { int }, N}^{\varepsilon}\right\|_{L^{2}}\left\|u^{\varepsilon}\right\|_{L^{2}} \int_{0}^{1} \mathrm{~d}\left(x_{3}\right)^{2}\left\|\partial_{3}\left(u_{\mathrm{app}, N}^{\varepsilon}-u_{0, \text { int }, N}^{\varepsilon}\right)\left(\cdot, x_{3}\right)\right\|_{L^{\infty}\left(\mathbf{R}^{2}\right)}^{2} \mathrm{~d} x_{3} .
$$


The estimate (2.10) allows to conclude that

$$
\int_{0}^{\infty}\left|\left(F_{N, 12}^{\varepsilon, 3}(t) \mid u^{\varepsilon}(t)\right)_{L^{2}}\right| \mathrm{d} t \leq C_{N^{\varepsilon^{2}}}{ }^{\frac{1}{2}}\left\|u_{0}\right\|_{L^{2}}^{2}
$$

So we have that

$$
\int_{0}^{\infty}\left|\left(F_{N, 1}^{\varepsilon}(t) \mid u^{\varepsilon}(t)\right)_{L^{2}}\right| \mathrm{d} t \leq C_{N} \varepsilon^{\frac{1}{2}}\left\|u_{0}\right\|_{L^{2}}^{2}
$$

Let us notice that no dispersion effects have been used to prove this estimate. But we shall use them to estimate

$$
\int_{0}^{\infty}\left|\left(F_{N, 2}^{\varepsilon}(t) \mid u^{\varepsilon}(t)\right)_{L^{2}}\right| \mathrm{d} t
$$

As $\bar{u}_{N}^{3}=0$, let us write that

$$
\begin{aligned}
& F_{N, 2}^{\varepsilon}=F_{N, 21}^{\varepsilon}+F_{N, 22}^{\varepsilon} \quad \text { with } \\
& F_{N, 21}^{\varepsilon} \stackrel{\text { def }}{=} \sum_{j=1}^{2} \partial_{j}\left(u_{0, \text { int }, N}^{\varepsilon, j} u_{0, \text { int }, N}^{\varepsilon}-\bar{u}_{N}^{j} \bar{u}_{N}\right) \text { and } \\
& F_{N, 22}^{\varepsilon} \stackrel{\text { def }}{=} \sum_{j=1}^{2} \partial_{3}\left(\left(u_{0, \text { int }, N}^{\varepsilon, 3}-\bar{u}_{N}^{3}\right) u_{0, \text { int }, N}^{\varepsilon}\right)
\end{aligned}
$$

As $u_{0, \text { int, } N}^{\varepsilon}$ is a (finite) sum of products of a function of the horizontal variable those Fourier transform is included in the ball of center 0 and radius $N$ by a function $\cos \left(k \pi x_{3}\right)$ or $\sin \left(k \pi x_{3}\right)$ we have, for $j \in\{1,2\}$,

$$
\left\|F_{N, 2 j}^{\varepsilon}(t)\right\|_{L^{2}} \leq C_{N}\left\|\left(u_{0, \text { int }, N}^{\varepsilon}-\bar{u}_{N}\right)(t)\right\|_{L^{\infty}}\left(\left\|u_{0, \text { int }, N}^{\varepsilon}(t)\right\|_{L^{2}}+\left\|\bar{u}_{N}(t)\right\|_{L^{\infty}}\right) .
$$

So using Lemma 6 and of course the conservation of energy for $(N S E)$ and the fact that $\mathcal{L}(\tau)$ is uniformly bounded on $L^{2}$, we can write that

$$
\int_{0}^{\infty}\left|\left(F_{N, 2}^{\varepsilon}(t) \mid u^{\varepsilon}(t)\right)_{L^{2}}\right| \mathrm{d} t \leq C_{N}\left\|u_{0, \text { int }, N}^{\varepsilon}-\bar{u}_{N}\right\|_{L^{1}\left(\mathbf{R}^{+} ; L^{\infty}\right)}\left\|u_{0}\right\|_{L^{2}}^{2} \leq C_{N} \varepsilon^{\frac{1}{4}}\left\|u_{0}\right\|_{L^{2}}^{2} .
$$

Lemma 5 is now proved and thus Theorem 2 also.

\section{Proof of the Strichartz type inequality}

In this final section, we shall prove Lemma 6, stated in the previous section. This type of estimate in the case of rotating fluids has been investigated in [3] and [4] in various situations. For the convenience of the reader, we give here a self contained proof. Thanks to the definition of $\mathcal{L}$ (see (2.4)) we have that

$$
\begin{gathered}
u_{0, \text { int }, N}^{\varepsilon}-\bar{u}_{N}=\sum_{k=1}^{N}\left(A_{k} \star \mathcal{I}_{k}^{\varepsilon}\left(t, x_{h}\right) \cos \left(k \pi x_{3}\right),-\frac{1}{k \pi} \operatorname{div}_{h} A_{k, N} \star \mathcal{I}_{k}^{\varepsilon}\left(t, x_{h}\right) \sin \left(k \pi x_{3}\right)\right) \text { with } \\
\mathcal{I}_{k}^{\varepsilon}\left(t, x_{h}\right) \stackrel{\text { def }}{=} \int_{\mathbf{R}^{2}} \mathrm{e}^{i\left(x_{h} \mid \xi_{h}\right)+i \frac{k \pi}{\left((k \pi)^{2}+\left|\xi_{h}\right|^{2}\right)^{1 / 2}} \frac{t}{\varepsilon}-\nu t\left|\xi_{h}\right|^{2}} \widehat{\gamma}_{k}\left(\xi_{h}\right) \mathrm{d} \xi_{h}
\end{gathered}
$$

where $A_{k}$ is a $L^{1}$ function on $\mathbf{R}^{2}$ and $\gamma_{k}$ is a linear function of $\widehat{d}_{k}$ and $\widehat{\omega}_{k}$. Now let $\varphi_{N}$ be a radial function 
in $\mathcal{D}\left(\mathbf{R}^{2} \backslash\{0\}\right)$ whose value is 1 near $\mathcal{C}_{N}$. We have

$$
\begin{aligned}
\mathcal{I}_{k}^{\varepsilon} & =\mathcal{K}_{k}\left(\frac{t}{\varepsilon}, t, \cdot\right) \star \gamma_{k} \text { with } \\
\mathcal{K}_{k}\left(\tau, t, x_{h}\right) & \stackrel{\text { def }}{=} \int_{\mathbf{R}^{2}} \mathrm{e}^{i\left(x_{h} \mid \xi_{h}\right)+i \frac{k \pi}{\left((k \pi)^{2}+\left|\xi_{h}\right|^{2}\right)^{1 / 2}} \tau-\nu t\left|\xi_{h}\right|^{2}} \varphi_{N}\left(\xi_{h}\right) \mathrm{d} \xi_{h} .
\end{aligned}
$$

As usual in the proof of Strichartz type estimates, we start by proving a so called dispersive estimate which here is the following:

Lemma 8. There exist constants $C_{N}$ and $c_{N}$ such that for any $k \leq N$, we have

$$
\left\|\mathcal{K}_{k}(\tau, t, \cdot)\right\|_{L^{\infty}\left(\mathbf{R}^{2}\right)} \leq \frac{C_{N}}{\tau^{\frac{1}{2}}} \mathrm{e}^{-c_{N} t} .
$$

Let us prove Lemma 8. Using the rotation invariance on $\mathbf{R}^{2}$, we may assume that $x_{h}=\left(x_{1}, 0\right)$. So we have to estimate

$$
\mathcal{K}_{k}\left(\tau, t, x_{1}, 0\right)=\int_{\mathbf{R}} \mathrm{e}^{i x_{1} \xi_{1}} \int_{\mathbf{R}} \mathrm{e}^{i \frac{k \pi}{\left((k \pi)^{2}+\xi_{1}^{2}+\xi_{2}^{2}\right)^{1 / 2}} \tau-\nu t\left(\xi_{1}^{2}+\xi_{2}^{2}\right)} \varphi_{N}\left(\xi_{1}, \xi_{2}\right) \mathrm{d} \xi_{2} \mathrm{~d} \xi_{1} .
$$

Now let us define the function $\alpha_{k}$ by

$$
\alpha_{k}\left(\xi_{2}\right) \stackrel{\text { def }}{=} \partial_{\xi_{2}} \frac{k \pi}{\left((k \pi)^{2}+\xi_{1}^{2}+\xi_{2}^{2}\right)^{1 / 2}}=\frac{\xi_{2} k \pi}{\left((k \pi)^{2}+\xi_{1}^{2}+\xi_{2}^{2}\right)^{3 / 2}} .
$$

We notice that

$$
\left|\alpha_{k}\left(\xi_{2}\right)\right| \geq C_{N}\left|\xi_{2}\right|
$$

Similarly we define the vector field $X_{k}$ by

$$
X_{k} a \stackrel{\text { def }}{=} \frac{1}{1+\tau \alpha_{k}^{2}\left(\xi_{2}\right)}\left(1+i \alpha_{k}\left(\xi_{2}\right) \partial_{\xi_{2}} a\right) .
$$

This vector field acts only on the $\xi_{2}$ variable and satisfies obviously

$$
X_{k} \mathrm{e}^{i \tau \frac{k \pi}{\left((k \pi)^{2}+\xi_{1}^{2}+\xi_{2}^{2}\right)^{1 / 2}}}=\mathrm{e}^{i \tau \frac{k \pi}{\left((k \pi)^{2}+\xi_{1}^{2}+\xi_{2}^{2}\right)^{1 / 2}}} .
$$

An integration by parts allows us to obtain

$$
\mathcal{K}_{k}\left(\tau, t, x_{1}, 0\right)=\int_{\mathbf{R}} \mathrm{e}^{i x_{1} \xi_{1}} \int_{\mathbf{R}} \mathrm{e}^{i \frac{k \pi}{\left((k \pi)^{2}+\xi_{1}^{2}+\xi_{2}^{2}\right)^{1 / 2} \tau}}\left({ }^{t} X_{k}\right)\left(\varphi_{N}\left(\xi_{1}, \xi_{2}\right) \mathrm{e}^{-\nu t\left(\xi_{1}^{2}+\xi_{2}^{2}\right)}\right) \mathrm{d} \xi_{2} \mathrm{~d} \xi_{1} .
$$

But easy computations yield

$$
\begin{array}{r}
{ }^{t} X_{k}\left(\varphi_{N}\left(\xi_{1}, \xi_{2}\right) \mathrm{e}^{-\nu t\left(\xi_{1}^{2}+\xi_{2}^{2}\right)}\right)=\left(\frac{1}{1+\tau \alpha_{k}^{2}\left(\xi_{2}\right)}-i\left(\partial_{\xi_{2}} \alpha_{k}\right) \frac{1-\tau \alpha_{k}^{2}\left(\xi_{2}\right)}{\left(1+\tau \alpha_{k}^{2}\left(\xi_{2}\right)\right)^{2}}\right) \\
-\frac{i \alpha_{k}\left(\xi_{2}\right)}{1+\tau \alpha_{k}^{2}\left(\xi_{2}\right)} \partial_{\xi_{2}}\left(\varphi_{N}\left(\xi_{1}, \xi_{2}\right) \mathrm{e}^{-\nu t\left(\xi_{1}^{2}+\xi_{2}^{2}\right)}\right) .
\end{array}
$$


Using (4.1), it turns out that

$$
\left|{ }^{t} X_{k}\left(\varphi_{N}\left(\xi_{1}, \xi_{2}\right) \mathrm{e}^{-\nu t\left(\xi_{1}^{2}+\xi_{2}^{2}\right)}\right)\right| \leq C_{N} \frac{\mathrm{e}^{-c_{N} t}}{1+\tau\left|\xi_{2}\right|^{2}}
$$

so going back to $\mathcal{K}_{k}$, we get

$$
\begin{aligned}
\left|\mathcal{K}_{k}\left(\tau, t, x_{h}\right)\right| & \leq C_{N} \mathrm{e}^{-c_{N} t} \int_{\mathcal{C}_{N}} \frac{\mathrm{d} \xi_{1} \mathrm{~d} \xi_{2}}{1+\tau\left|\xi_{2}\right|^{2}} \\
& \leq \frac{C_{N}}{\tau^{1 / 2}} \mathrm{e}^{-c_{N} t}
\end{aligned}
$$

which proves Lemma 8 .

Now let us finish the proof of Lemma 6 . As an immediate corollary of the result above, we have

$$
\left\|\mathcal{K}_{k}\left(\frac{t}{\varepsilon}, t, \cdot\right)\right\|_{L^{\infty}\left(\mathbf{R}^{2}\right)} \leq \frac{C_{N} \varepsilon^{1 / 2}}{t^{1 / 2}} \mathrm{e}^{-c_{N} t}\left\|\gamma_{k}\right\|_{L^{1}\left(\mathbf{R}^{2}\right)}
$$

Now we shall use a duality argument, otherwise known as the $T T^{*}$ argument. Once we have observed that

$$
\|a\|_{L^{1}\left(\mathbf{R}^{+} ; L^{\infty}\left(\mathbf{R}^{2}\right)\right)}=\sup _{\varphi \in \mathcal{B}} \int_{\mathbf{R}^{+} \times \mathbf{R}^{2}} a\left(t, x_{h}\right) \varphi\left(t, x_{h}\right) \mathrm{d} x_{h} \mathrm{~d} t
$$

with $\mathcal{B} \stackrel{\text { def }}{=}\left\{\varphi \in \mathcal{D}\left(\mathbf{R}^{+} \times \mathbf{R}^{2}\right),\|\varphi\|_{L^{\infty}\left(\mathbf{R}^{+} ; L^{1}\left(\mathbf{R}^{2}\right)\right)} \leq 1\right\}$, we can write

$$
\begin{aligned}
\| \mathcal{K}_{k}\left(\frac{t}{\varepsilon}, t, \cdot\right) & * \gamma_{k} \|_{L^{1}\left(\mathbf{R}^{+} ; L^{\infty}\left(\mathbf{R}^{2}\right)\right)}=\sup _{\varphi \in \mathcal{B}} \int_{\mathbf{R}^{+} \times \mathbf{R}^{4}} \mathcal{K}_{k}\left(\frac{t}{\varepsilon}, t, x-y\right) \gamma_{k}(y) \varphi(t, x) \mathrm{d} x \mathrm{~d} y \mathrm{~d} t \\
& =\sup _{\varphi \in \mathcal{B}} \int_{\mathbf{R}^{+} \times \mathbf{R}^{2}} \gamma_{k}(y)\left(\int_{\mathbf{R}^{2}} \mathcal{K}_{k}\left(\frac{t}{\varepsilon}, t, x-y\right) \varphi(t, x) \mathrm{d} x\right) \mathrm{d} y \mathrm{~d} t .
\end{aligned}
$$

A Cauchy-Schwarz inequality then yields

$$
\left\|\mathcal{K}_{k}\left(\frac{t}{\varepsilon}, t, \cdot\right) * \gamma_{k}\right\|_{L^{1}\left(\mathbf{R}^{+} ; L^{\infty}\left(\mathbf{R}^{2}\right)\right)} \leq\left\|\gamma_{k}\right\|_{L^{2}\left(\mathbf{R}^{2}\right)} \sup _{\varphi \in \mathcal{B}}\left\|\int_{\mathbf{R}^{+}} \check{\mathcal{K}}_{k}\left(\frac{t}{\varepsilon}, t, \cdot\right) * \varphi_{k}(t, \cdot) \mathrm{d} t\right\|_{L^{2}\left(\mathbf{R}^{2}\right)} .
$$

By Fourier-Plancherel, we have

$$
\left\|\int_{\mathbf{R}^{+}} \check{\mathcal{K}}_{k}\left(\frac{t}{\varepsilon}, t, \cdot\right) * \varphi(t, \cdot) \mathrm{d} t\right\|_{L^{2}\left(\mathbf{R}^{2}\right)}^{2}=(2 \pi)^{2}\left\|\int_{\mathbf{R}^{+}} \overline{\widehat{\mathcal{K}}_{k}}\left(\frac{t}{\varepsilon}, t, \cdot\right) \widehat{\varphi}(t, \cdot) \mathrm{d} t\right\|_{L^{2}\left(\mathbf{R}^{2}\right)}^{2}
$$

and we can write

$$
\left\|\int_{\mathbf{R}^{+}} \overline{\widehat{\mathcal{K}}_{k}}\left(\frac{t}{\varepsilon}, t, \cdot\right) * \widehat{\varphi}(t, \cdot) \mathrm{d} t\right\|_{L^{2}\left(\mathbf{R}^{2}\right)}^{2} \leq C \int_{\mathbf{R}^{+} \times \mathbf{R}^{+} \times \mathbf{R}^{2}} \widehat{\mathcal{K}}_{k}\left(\frac{t}{\varepsilon}, t,-\xi_{h}\right) \widehat{\varphi}\left(t, \xi_{h}\right) \overline{\widehat{\mathcal{K}}_{k}}\left(\frac{s}{\varepsilon}, s, \xi_{h}\right) \widehat{\varphi}\left(s, \xi_{h}\right) \mathrm{d} \xi_{h} \mathrm{~d} t \mathrm{~d} s
$$

But by definition of $\mathcal{K}$, we have

$$
\widehat{\mathcal{K}}_{k}\left(\frac{t}{\varepsilon}, t,-\xi_{h}\right) \overline{\mathcal{K}}_{k}\left(\frac{s}{\varepsilon}, s, \xi_{h}\right)=\widehat{\mathcal{K}}_{k}\left(\frac{t-s}{\varepsilon}, t+s,-\xi_{h}\right) .
$$


It follows that

$$
\left\|\int_{\mathbf{R}^{+}} \check{\mathcal{K}}_{k}\left(\frac{t}{\varepsilon}, t, \cdot\right) * \varphi(t, \cdot) \mathrm{d} t\right\|_{L^{2}\left(\mathbf{R}^{2}\right)}^{2} \leq C \int_{\mathbf{R}^{+} \times \mathbf{R}^{+} \times \mathbf{R}^{2}} \mathcal{F}\left(\check{\mathcal{K}}_{k}\left(\frac{t-s}{\varepsilon}, t+s, \cdot\right) * \varphi(t, \cdot)\right) \overline{\widehat{\varphi}}\left(s, \xi_{h}\right) \mathrm{d} \xi_{h} \mathrm{~d} t \mathrm{~d} s .
$$

Now we use Fourier-Plancherel again to get

$$
\begin{aligned}
\left\|\int_{\mathbf{R}^{+}} \check{\mathcal{K}}_{k}\left(\frac{t}{\varepsilon}, t, \cdot\right) * \varphi(t, \cdot) \mathrm{d} t\right\|_{L^{2}\left(\mathbf{R}^{2}\right)}^{2} & \leq C \int_{\mathbf{R}^{+} \times \mathbf{R}^{+} \times \mathbf{R}^{2}}\left(\check{\mathcal{K}}_{k}\left(\frac{t-s}{\varepsilon}, t+s, \cdot\right) * \varphi(t, \cdot)\right)(x) \varphi(s,-x) \mathrm{d} x \mathrm{~d} t \mathrm{~d} s \\
& \leq C \int_{\mathbf{R}^{+} \times \mathbf{R}^{+}}\left\|\check{\mathcal{K}}_{k}\left(\frac{t-s}{\varepsilon}, t+s, \cdot\right) * \varphi(t, \cdot)\right\|_{L^{\infty}\left(\mathbf{R}^{2}\right)}\|\varphi(s, \cdot)\|_{L^{1}\left(\mathbf{R}^{2}\right)} \mathrm{d} t \mathrm{~d} s
\end{aligned}
$$

Using the dispersion estimate (4.2), we get

$$
\left\|\int_{\mathbf{R}^{+}} \check{\mathcal{K}}_{k}\left(\frac{t}{\varepsilon}, t, \cdot\right) * \varphi(t, \cdot) \mathrm{d} t\right\|_{L^{2}\left(\mathbf{R}^{2}\right)}^{2} \leq C_{N} \int_{\mathbf{R}^{+} \times \mathbf{R}^{+}} \frac{\varepsilon^{1 / 2}}{(t-s)^{1 / 2}} \mathrm{e}^{-c_{N}(t+s)}\|\varphi(t, \cdot)\|_{L^{1}\left(\mathbf{R}^{2}\right)}\|\varphi(s, \cdot)\|_{L^{1}\left(\mathbf{R}^{2}\right)} \mathrm{d} s \mathrm{~d} t
$$

SO

$$
\begin{aligned}
\left\|\int_{\mathbf{R}^{+}} \check{\mathcal{K}}_{k}\left(\frac{t}{\varepsilon}, t, \cdot\right) * \varphi(t, \cdot) \mathrm{d} t\right\|_{L^{2}\left(\mathbf{R}^{2}\right)}^{2} & \leq C_{N} \varepsilon^{1 / 2}\|\varphi\|_{L^{\infty}\left(\mathbf{R}^{+} ; L^{1}\left(\mathbf{R}^{2}\right)\right)}^{2} \int_{\mathbf{R}^{+} \times \mathbf{R}^{+}} \frac{\mathrm{e}^{-c_{N}(t+s)}}{(t-s)^{1 / 2}} \mathrm{~d} s \mathrm{~d} t \\
& \leq C_{N} \varepsilon^{1 / 2}\|\varphi\|_{L^{\infty}\left(\mathbf{R}^{+} ; L^{1}\left(\mathbf{R}^{2}\right)\right)}^{2} .
\end{aligned}
$$

Lemma 6 is proved.

\section{REFERENCES}

[1] A. Babin, A. Mahalov and B. Nicolaenko, Global regularity of 3D rotating Navier-Stokes equations for resonant domains. Indiana Univ. Math. J. 48 (1999) 1133-1176.

[2] A. Babin, A. Mahalov and B. Nicolaenko, Global splitting, integrability and regularity of 3D Euler and Navier-Stokes equations for uniformly rotating fluids. European J. Mech. B Fluids 15 (1996) 291-300.

[3] J.-Y. Chemin, B. Desjardins, I. Gallagher and E. Grenier, Fluids with anisotropic viscosity. Modél. Math. Anal. Numér. 34 (2000) 315-335.

[4] J.-Y. Chemin, B. Desjardins, I. Gallagher and E. Grenier, Anisotropy and dispersion in rotating fluids. Preprint of Orsay University.

[5] B. Desjardins, E. Dormy and E. Grenier, Stability of mixed Ekman-Hartmann boundary layers. Nonlinearity 12 (1999) 181-199.

[6] I. Gallagher, Applications of Schochet's methods to parabolic equations. J. Math. Pures Appl. 77 (1998) 989-1054.

[7] H.P. Greenspan, The theory of rotating fluids, Reprint of the 1968 original. Cambridge University Press, Cambridge-New York, Cambridge Monogr. Mech. Appl. Math. (1980).

[8] E. Grenier, Oscillatory perturbations of the Navier-Stokes equations. J. Math. Pures Appl. 76 (1997) 477-498.

[9] E. Grenier and N. Masmoudi, Ekman layers of rotating fluids, the case of well prepared initial data. Comm. Partial Differential Equations 22 (1997) 953-975.

[10] N. Masmoudi, Ekman layers of rotating fluids: The case of general initial data. Comm. Pure Appl. Math. 53 (2000) $432-483$.

[11] Pedlovsky, Geophysical Fluid Dynamics. Springer-Verlag (1979). 